\title{
Quasiclassical Molecular Dynamics Simulation of the Photoisomerization of Stilbene
}

\author{
Valentin D. Vachev and John H. Frederick* \\ Department of Chemistry, University of Nevada, Reno, Nevada 89557 \\ Boris A. Grishanin, ${ }^{\dagger}$ Victor N. Zadkov, ${ }^{\ddagger}$ and Nikolai I. Koroteev ${ }^{\dagger, \ddagger}$ \\ Physics Department and International Laser Center, Moscow State University, 119899 Moscow, Russia
}

Received: November 2, 1994; In Final Form: January 24, $1995^{\circledR}$

The ultrafast laser-induced isomerization dynamics of gas phase stilbene is studied in detail using quasiclassical molecular dynamics methods. To model the dynamics, empirical potential surfaces for both the ground and the first excited electronic states of stilbene are constructed using molecular modeling-type potentials which are fit to available spectroscopic measurements, ultrafast dynamics observations, and theoretical structural information. An algorithm for creating quasiclassical initial conditions that simulate the nonstationary state prepared by an ultrashort (100 fs) laser pulse is presented. This algorithm ultilizes a quantum mechancial formulation of the excitation based on harmonic approximations for the potential surfaces which is then adapted to give initial conditions for an ensemble of trajectories. Using these methods, we recently (Chem. Phys. Lett. 1993, 215, 306) found evidence for the existence of a small barrier along the cis-trans isomerization (ethylenic torsion) coordinate from the cis-stilbene Franck-Condon region of the excited state and here we improve the estimate of that barrier to between 260 and $400 \mathrm{~cm}^{-1}$. Detailed examination of the excited state dynamics of $c i s$-stilbene reveals that the isomerization process proceeds via multiple pathways to a twisted potential minimum on the excited state from which internal conversion to the ground state occurs. All three photoproducts of $c i s$-stilbene cross to the ground state from the same general region of the excited state and are differentiated during the radiationless transition and subsequent dynamics on the ground state. Nonadiabatic coupling between the electronic states is included in our studies using a semiclassical technique, and this allows the identification of correlations between the excited state dynamics and the branching between final products. The photoexcitation of trans-stilbene is also examined, and our calculations suggest that this isomerization process proceeds through a different minimum on the excited state surface which also corresponds to a $90^{\circ}$ twisted configuration. This is a departure from the standard view that both cis- and trans-stilbene proceed through the same intermediate in their respective cis-trans photoisomerization dynamics.

\section{Introduction}

A detailed microscopic understanding of intramolecular dynamics and reaction mechanisms is a necessary prerequisite for exercising control over the outcome of a chemical reaction. ${ }^{1.2}$ Dynamical understanding aids not only in the study of complicated natural processes such as photoinduced isomerization in retinal systems and photosynthetic bacteria and electron and proton transfer in biological systems ${ }^{1,3}$ but also in the design of molecular switches (so-called "nanoswitches"), molecular electronics, and nonlinear materials for optoelectronics. ${ }^{4,5}$ The study of molecular dynamics has been greatly enhanced in recent years with the advent of both ultrashort laser pulses and nonlinear techniques for the generation of frequencies in a desired range. ${ }^{6.7}$ These techniques augment the wealth of data available from the precise determination of molecular frequencies and anharmonicities via high-resolution spectroscopy. In particular, experiments can now provide a microscopic picture of ultrafast molecular processes, including the interaction of a molecule with a short laser pulse, the mechanism for intramolecular vibrational energy redistribution (IVR), the evolution of a system through a transition state (TS) to final products, and finally the dissipation of energy into the environment. ${ }^{1,3}$

\footnotetext{
* Author to whom correspondence should be addressed.

+ Physics Department, Moscow State University.

† International Laser Center, Moscow State University.

\& Abstract published in Advance ACS Abstracts, March 15, 1995.
}

For more than four decades, stilbene (see Figure 1) has been a prototype molecule for studying photoinduced isomerization dynamics, both in condensed and gas phase environments. ${ }^{8}$ In recent years, a number of groups have used femtosecond time resolution to characterize the ultrafast photoisomerization dynamics of cis-stilbene, which undergoes competing reactions to form both cis- and trans-stilbene, as well as a photocyclization product, dihydrophenanthrene (DHP), when photoexcited. In the gas phase, cis-stilbene exhibits a decay time of $\approx 300 \mathrm{fs}$ in its first excited singlet state, ${ }^{9,10}$ compared with the much longer decay time of $80 \mathrm{ps}$ observed for isolated trans-stilbene. However, using a time resolution of $80 \mathrm{fs}$, Zewail and co-workers ${ }^{10}$ have resolved irregular oscillations imposed on the exponential decay curve and, while these are difficult to relate directly to information about the reaction coordinate, they appear to be a clear manifestation of nonstatistical decay behavior. This is also reflected in the observed increase of the excited state lifetime with increase in the excitation (photon) energy.

As a further probe of the nature of the excited state potential of cis-stilbene, Petek et al. ${ }^{11}$ have used ground state recovery experiments to measure the lifetime of the excited state in noble gas clusters, observing $\tau=1.7 \mathrm{ps}$ in $\mathrm{Ar}$ and $3.0 \mathrm{ps}$ in $\mathrm{Kr}$. Those workers also observe long-lived fluorescence $(\approx 20 \mathrm{~ns})$ in these complexes which they interpret as strong evidence for a local minimum in the cis-stilbene configuration of the cluster and for the importance of vibrational cooling from the environment. 


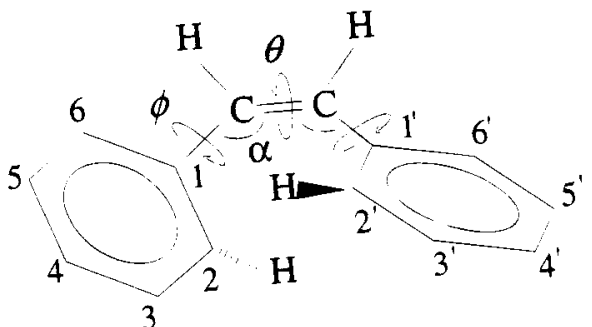

Figure 1. cis-Stilbene structure. In the present study, all 72 vibrations are included, although the analysis centers on torsional $(\theta)$ and bending $(\alpha)$ angles, as well as the $\mathrm{C}_{2}-\mathrm{C}_{2}$ ' distance which is directly associated with photocyclization

On the basis of 1,2-diphenylcyclobutene spectral assignments and the similarity between the nonradiative decays of cis-stilbene and 1,2-diphenylcyclopentene, Petek and co-workers ${ }^{12}$ have proposed that the initial motion on the $S_{1}$ surface of $c i s$-stilbene proceeds along the reaction coordinate for photocyclization rather than along the torsional (i.e., cis-trans) isomerization coordinate and emphasize the importance of considering the DHP isomerization channel in cis-stilbene excited state dynamics.

More extensive studies have explored the photoisomerization of cis-stilbene in liquids, ${ }^{13-20}$ probing the effects of a solvent environment on both the rate and the outcome of the reaction, as well as the details of the reaction mechanism. Decay times have been determined in a variety of solvents and range from $0.38 \mathrm{ps}$ in acetonitrile and $0.5 \mathrm{ps}$ in methanol to $1.83 \mathrm{ps}$ in cyclohexane. These decay times generally fall in the range of $0.5 \mathrm{ps}$ in alcohols and from about 1 to $2 \mathrm{ps}$ in alkenes with no spectral evolution during the lifetime, and similar results are obtained from both transient absorption and fluorescence spectroscopy. Todd et al. ${ }^{13}$ and Rice and Baronavski ${ }^{14}$ have compared excited state lifetime data in both polar and nonpolar solvents and concluded that microscopic solvent-solute interactions are present and their contributions are larger than those due to the bulk viscosity dependencies. In related studies, Troe and co-workers ${ }^{15}$ have varied the viscosity in a single solvent by changing the pressure and/or temperature in order to separate static and dynamical effects. They find a linear dependence for the decay times with the inverse of the viscosity and attribute the different slopes obtained for different solvents to changes in the potential energy surface for cis-trans isomerization. Todd and Fleming ${ }^{16}$ have suggested an alternative interpretation for the linear dependencies, proposing that the slopes indicate the relationship between macroscopic viscosity and the friction experienced by isomerizing cis-stilbene. They correlated the decay times in $n$-alcohols and $n$-alkanes to the chain length and concluded that for $n \geq 5$, the potential surface felt by stilbene is not substantially altered.

In related work, Hochstrasser's group has studied the photoisomerization of cis-stilbene by observing both the formation of the product molecules and the time evolution of the molecule on its excited state on an ultrafast time scale leading to a comprehensive dynamical picture of the reaction. Their experiments ${ }^{3.17-19}$ demonstrate that vibrationally hot product molecules are formed after the escape of the molecule from the cis*-region of the potential energy surface (here, this region is defined by a vertical Franck-Condon transition from ground state cis-stilbene). trans-Stilbene fluorescence was found to rise on the same time scale as that found for the disappearance of the $c i s^{*}$-population, thus restricting the time spent on the reaction pathway to the time resolution of the experiment, namely 150 fs. The DHP product molecules are found to appear in a few hundred fematoseconds with the lifetime of the intermediate being $300 \pm 200 \mathrm{fs}$. In addition, anisotropy measurements ${ }^{3.19}$ reveal that the orientation of the reactant-product dipoles are similar for the cis $\rightarrow$ trans $\left(35 \pm 4^{\circ}\right)$ and cis $\rightarrow$ DHP $\left(38 \pm 3^{\circ}\right)$ reactions in hexadecane, indicating a more complicated reaction coordinate for the DHP channel than simple electronic rearrangement and bond formation. Finally, Myers and coworkers ${ }^{20}$ have also monitored hot products (trans-stilbene) and find solvent dependence in the vibrational cooling.

Despite the wealth of available data and the variety of experimental observations that have been made, the mechanism of the photoisomerization reactions of cis-stilbene remains largely unknown. Because both static and dynamical effects are thought to play important roles in the solvation, the intramolecular dynamics and reaction mechanism in the isolated molecule must first be well understood in order to separate and understand the effects due to solvent interactions. In this paper, we present a comprehensive study of the excited state dynamics of isolated cis-stilbene, including all 72 vibrational degrees of freedom, using quasiclassical molecular dynamics (MD) simulation methods. Our study includes the construction of an empirical global $S_{1}$ state potential surface for stilbene, the classical simulation of the dynamics of an initial state prepared by an ultrashort laser pulse, and the semiclassical dynamics of internal conversion back to the ground electronic state. To optimize the qualitative accuracy of our empirical $S_{1}$ surface, its parameters have been adjusted to reproduce as many experimental observations and quantum calculations as possible. Besides the ultrafast time-resolved measurements discussed above, this parametrization is derived from (i) measurements of photochemical products from both cis- and trans-stilbene over a wide range in temperature and solvent viscosity,,$^{21-26}$ (ii) semiempirical electronic structure calculations, ${ }^{27-33}$ (iii) highresolution spectroscopy of trans-stilbene $e^{34-38}$ and diphenylcyclobutene, ${ }^{12}$ (iv) resonance Raman and CARS spectroscopy of stilbene and DHP, ${ }^{39-41}$ and (v) absorption/emission spectra calculations. ${ }^{42-44}$

A key issue in ths work is the characterization of the $S_{1}$ excited state surface. The two stable isomers are separated by a $48 \mathrm{kcal} / \mathrm{mol}\left(16800 \mathrm{~cm}^{-1}\right)$ barrier in the ground state $\left(\mathrm{S}_{0}\right)$ from the cis side, and the cis-stilbene minimum is $4.59 \mathrm{kcal} /$ mol $\left(1605 \mathrm{~cm}^{-1}\right)$ higher in energy than that for trans-stilbene. ${ }^{45}$ The spectral origins for the corresponding $S_{1}-S_{0}$ transitions are placed at $321.0 \mathrm{~nm}\left(31152.6 \mathrm{~cm}^{-1}\right)$ for trans and 343.5 $\mathrm{nm}\left(29112.0 \mathrm{~cm}^{-1}\right)$ for cis, both in Ar clusters, ${ }^{12}$ making cis* about $450 \mathrm{~cm}^{-1}$ lower in energy than trans* in $\mathrm{S}_{1 .}{ }^{10}$ A local barrier to cis-trans isomerization of about $1200 \mathrm{~cm}^{-1}$ from the trans* side in the isolated molecule is well established experimentally. ${ }^{46}$ It is also generally postulated that cis-trans isomerization starting from both cis- and trans-stilbene proceeds through a common intermediate $\left({ }^{1} \mathrm{p}^{*}\right)$ at the $90^{\circ}$ twisted configuration of the central ethylenic bond. ${ }^{8}$ The very short lifetime of the twisted intermediate $\left({ }^{1} \mathrm{p}^{*}\right)$ suggests a very small energy gap at this position; thus, the energy difference between the trans* and ' $\mathrm{p}$ * configurations has been estimated to be about $45 \mathrm{kcal} / \mathrm{mol}\left(15740 \mathrm{~cm}^{-1}\right)$. The overall shape of the potential along the reaction coordinate is believed to arise from a mixing of the first excited $B$ state (a $\pi \rightarrow \pi^{*}$ transition) with one or more higher lying, doubly excited configurations that have a minimum at the $90^{\circ}$ twisted form..$^{8 \mathrm{~b}}$

More recent quantum calculations indicate a preference for an adiabatic $S_{1}$ surface description. ${ }^{30.31}$ Using the CNDO/S method, Rettig et al. ${ }^{31}$ have shown that with a proper choice of molecular orbitals used for the configuration interaction, ground and singly excited configurations are sufficient for a description of the lowest excited states and that the lowest doubly excited state is considerably higher in energy for both planar and double 

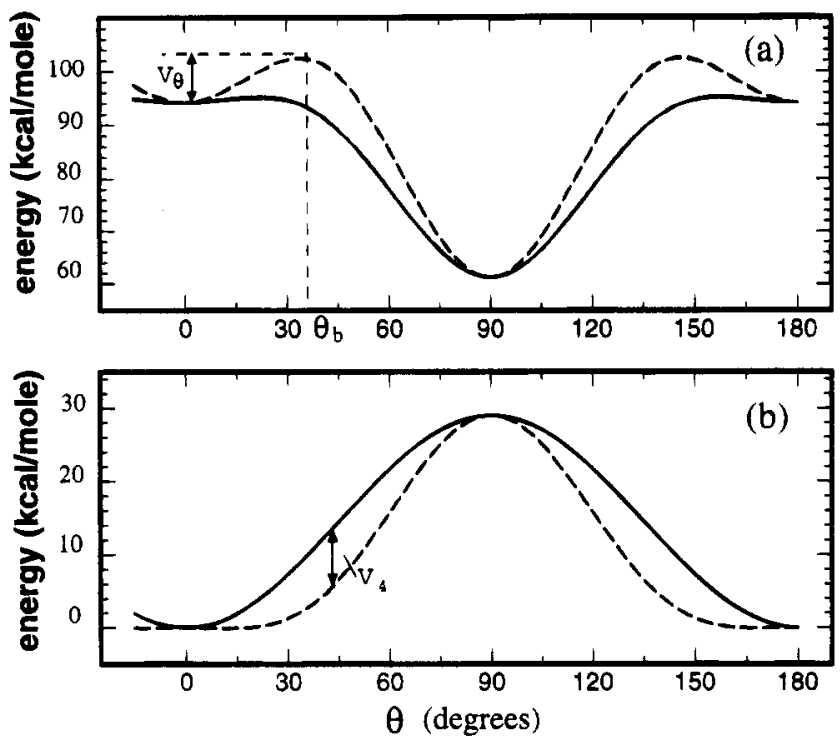

Figure 2. Torsional potentials (eq 17) with parameters representative for the excited (a) and ground (b) states of stilbene. Variation of the parameters $V_{2}$ and $V_{4}$ allows for modeling different barriers and shapes. In (a) $V_{2}<0$ and as $V_{4}$ increases (dashed line) the barrier becomes higher and its position moves to higher values of the torsional angle. In (b) $V_{2}>0$ and the region of the minimum becomes flatter as $V_{4}$ decreases (dashed line).

bond twisted configurations. They estimate the energy difference between $S_{0}$ and $S_{1}$ at $\theta=90^{\circ}$ to be $5.5 \mathrm{kcal} / \mathrm{mol}(1925$ $\mathrm{cm}^{-1}$ ) which is in accordance with experimental results and the energy gap rule. In a recent preliminary report of stilbene excited state dynamics, ${ }^{47}$ we have used classical MD simulations on an empirical $S_{1}$ surface without constraints to establish the existence of a barrier along the ethylenic torsion coordinate from the cis* side of between 510 and $640 \mathrm{~cm}^{-1}$ in the isolated molecule. This barrier is illustrated in Figure 2 where we show plots of the ground and excited state potential energy surfaces along the torsional coordinate.

Theoretical calculations also predict that the bond order of $\mathrm{C}_{\mathrm{e}}=\mathrm{C}_{\mathrm{e}^{\prime}}$ decreases and $\mathrm{C}_{1}-\mathrm{C}_{\mathrm{e}}$ increases for $\pi^{*} \leftarrow \pi$ excitation to the $S_{1}$ state of stilbene. ${ }^{27-33}$ This has the effect of increasing the $C_{e}=C_{e^{\prime}}$ bond length and decreasing the $C_{1}-C_{e}$ bond lengths. The changes in the electronic structure of the $\pi$-system also increase the torsion $(\theta)$ angle and decrease the bend $(\alpha)$ and twist $(\phi)$ angles in the $S_{1}$ state. Theoretical and experimental evidence have been prosented, ${ }^{27,48}$ for a $\sigma$-bonding interaction between carbon atoms at the 2 and $2^{\prime}$ positions in the $S_{1}$ state (as labeled in Figure 1) which is responsible for the observed photocyclization channel. Frederick et al. ${ }^{12 \mathrm{~b}}$ have suggested that the twist coordinate $(\phi)$ may play a major role in opening this channel because $\phi$ is strongly activated upon photoexcitation and, along with the bending coordinate $\alpha$, can mediate the interplay between the $\mathrm{C}_{2}-\mathrm{C}_{2^{\prime}}$ attractive interaction and the $\mathrm{H}_{2}-$ $\mathrm{H}_{2}$, repulsion. In other words, the relative orientation of the two phenyl rings is as important as their distance apart in activating the photocyclization channel.

In this work we extend our earlier study of cis-stilbene excited state dynamics ${ }^{47}$ by both improving the estimate for the local barrier along the torsional coordinate and including the nonadiabatic coupling with the ground state in the model. In this way, the current model allows the investigation of the various dynamical pathways ror formation of DHP and trans-stilbene from excited cis-stilhene. In section II, we describe the construction of our model empirical potential energy surfaces using spectral calculations and our quasiclassical methodology for simulating the initial wave packet prepared by an ultrashort pulse of finite duration absorbed by a sample at finite temperature. Our results for the excited state dynamics of cis-stilbene are presented in section III where we analyze the dynamics of a large ensemble of trajectories as a model for a nonstationary quantum state. A dynamical picture of the formation of products is considered in section IV; and in section V we summarize our results and discuss their implications.

\section{Spectra Calculation and Model Potential Energy Surfaces}

Modeling laser-initiated dynamics in molecules the size of stilbene requires appropriate approximations. In particular, two major theoretical challenges include (i) the construction of suitable Born-Oppenheimer potential surfaces on which the dynamics is to evolve and (ii) the propagation of nonstationary quantum states in all 72 vibrational degrees of freedom. Despite impressive advances in the capabilities of $a b$ initio methods in recent years, the calculation of a full ground state potential energy surface remains prohibitive for a molecule such as stilbene, much less the characterization of an excited state surface. For this reason, we utilize empirical "molecular modeling"-type potentials in the present studies whose parameters are adjusted to reproduce spectroscopic observations. As much as possible, relevant information from electronic structure calculations is incorporated into these surfaces, including structural information about bond lengths and angles and guidelines about how the structure changes upon excitation to the $S_{1}$ state. To explore the dynamics, we use classical molecular dynamics simulations to follow propagation on the excited state. Here, the initial conditions are determined by applying the quantum density matrix formulation of photoexcitation by an ultrashort laser pulse, and the dynamics of an ensemble of classical trajectories on empirical potential surfaces is analyzed to simulate the full dynamics of a quantum wave packet. The details of our treatment are given below.

Spectra Calculations. If we consider a harmonic polyatomic molecule with two adiabatic electronic states $|\mathrm{g}\rangle$ and $|\mathrm{e}\rangle$ and $N_{\mathrm{v}}$ vibrational degrees of freedom, we can write the Hamiltonian (in the absence of an electromagnetic field and electronic relaxation) as

$$
\mathscr{H}=|\mathrm{g}\rangle \mathscr{K} \mathrm{g}\langle\mathrm{g}|+| \mathrm{e}\rangle\left(\hbar \omega_{\mathrm{ge}}+\mathscr{H} \mathrm{e}\langle\mathrm{e}|\right.
$$

with

$$
\begin{aligned}
& \mathscr{K}_{\mathrm{g}}=\frac{\hbar}{2} \sum_{j=1}^{N_{\mathrm{v}}} \omega_{j}^{\prime \prime}{ }_{j}\left(\tilde{p}^{\prime \prime 2}{ }_{j}^{2}+\tilde{q}_{j}^{\prime 2}-1\right), \\
& \mathscr{K}_{\mathrm{e}}=\frac{\hbar}{2} \sum_{j=1}^{N_{\mathrm{v}}} \omega_{j}^{\prime}\left(\tilde{p}_{j}^{\prime 2}+\tilde{q}_{j}^{\prime 2}-1\right)
\end{aligned}
$$

and where $\tilde{\mathbf{q}}^{\prime}$ and $\tilde{\mathbf{q}}^{\prime \prime}$ are related by

$$
\tilde{\mathbf{q}}^{\prime}=\mathbf{S} \tilde{\mathbf{q}}^{\prime \prime}+\overline{\mathbf{D}}
$$

Here, $\omega_{\mathrm{ge}}$ is the electronic transition frequency between the lowest vibronic states of $|\mathrm{g}\rangle$ and $|\mathrm{e}\rangle(0-0$ transition). For convenience we have adopted the notation used by $Y$ an and Mukamel, ${ }^{49}$ and we label ground state quantities by a double prime and excited state quantities by a single prime. In eqs 1 and $2, \tilde{\mathbf{p}}^{\prime}$ and $\tilde{\mathbf{q}}^{\prime}$ denote the dimensionless normal mode momentum and position vectors, respectively, on the excited state. Likewise, $\tilde{\mathbf{p}}^{\prime \prime}$ and $\tilde{\mathbf{q}}^{\prime \prime}$ represent dimensionless normal mode momentum and position vectors on the ground state. $\mathbf{S}$ is 
the Dushinsky rotation matrix and $\overline{\mathbf{D}}$ is the vector of dimensionless displacements between the electronic surfaces.

These vectors can be related to the dimensional Cartesian displacement and momentum coordinates, $(\mathbf{r}, \mathbf{p})$, using the normal mode transformation for mass-weighted coordinates on the ground state. This gives (in matrix notation)

$$
\begin{gathered}
\tilde{\mathbf{p}}^{\prime \prime}=\frac{1}{\hbar^{1 / 2}} \mathbf{\Omega}^{\prime \prime-1 / 2} \cdot \mathbf{V} \cdot \mathbf{M}^{-1 / 2} \cdot \mathbf{p}, \\
\tilde{\mathbf{q}}^{\prime \prime}=\frac{1}{\hbar^{1 / 2}} \mathbf{\Omega}^{\prime \prime 1 / 2} \cdot \mathbf{V} \cdot \mathbf{M}^{1 / 2} \cdot \mathbf{r}
\end{gathered}
$$

where $\boldsymbol{\Omega}^{\prime \prime}$ and $\mathbf{M}$ are diagonal matrices whose elements are the normal mode frequencies and atomic masses, respectively, and $\mathbf{V}$ is the diagonalizing unitary transformation matrix that relates the Cartesian displacements, $\mathbf{r}$, to the mass-weighted normal mode coordinates, $\mathbf{Q}^{\prime \prime}$, that is,

$$
\mathbf{Q}^{\prime \prime}=\mathbf{V} \cdot \mathbf{M}^{1 / 2} \cdot \mathbf{r}, \quad \mathbf{P}^{\prime \prime}=\mathbf{V} \cdot \mathbf{M}^{-1 / 2} \cdot \mathbf{p}
$$

We discard the six zero-frequency normal modes arising from translation and rotation to obtain $(3 N-6)$ normal mode coordinates.

In eq 2 , the Dushinsky rotation matrix, $\mathbf{S}$, allows the ground and excited state normal coordinates to be different. If the normal modes in the ground and the electronically excited states are identical, the Dushinsky transformation matrix is diagonal, that is,

$$
S_{i j}=\left(\frac{\omega_{i}^{\prime}}{\omega^{\prime \prime}}\right) \delta_{i j}
$$

and the transformation between the normal coordinates is

$$
\mathbf{Q}^{\prime}=\mathbf{Q}^{\prime \prime}+\mathbf{D}
$$

The components of the dimensionless displacement vector $\tilde{D}_{j}$ are related to the $D_{j}$ by

$$
\bar{D}_{j}=\left(\frac{\omega_{j}^{\prime}}{\hbar}\right)^{1 / 2} D_{j}
$$

So far we have considered an isolated molecule having two electronic states with excited state represented in the normal mode basis of the ground state. As a result, the electronicvibrational spectroscopy of the system can be characterized in a convenient way using the normal mode frequencies and the displacements along the normal coordinates. The electromagnetic field of a laser pulse couples the two electronic states through the dipole moment of the transition (under the dipole approximation). Utilizing the density matrix formalism ${ }^{50}$ and the interaction representation ${ }^{51}$ for the molecular system interacting with a laser field, the density matrix of the excited state is given by ${ }^{52}$

$$
\begin{aligned}
\hat{\varrho}_{\mathrm{e}}=\frac{\left|d_{\mathrm{ge}}\right|^{2}}{4 \hbar^{2}} \iint \mathrm{d} \tau_{1} \mathrm{~d} \tau_{2} E_{L}\left(\tau_{1}\right) E_{L}^{*}\left(\tau_{2}\right) \times \\
\exp \left[-\frac{\mathrm{i}}{\hbar} \hat{\mathscr{H}}_{\mathrm{e}}(t-s)\right] \hat{\varrho}_{\mathrm{e}}^{0}(\tau) \exp \left[\frac{\mathrm{i}}{\hbar} \hat{\mathscr{H}}_{\mathrm{e}}(t-s)\right]
\end{aligned}
$$

where we have used a second-order perturbation expansion with respect to the field and the Condon approximation for the electronic transition dipole moment, $d_{\mathrm{ge}}$. In this equation $E_{\mathrm{L}}\left(\tau_{k}\right)$ is the complex wave function of the laser field, $\tau=\tau_{2}$ $-\tau_{1} ; s=\left(\tau_{2}+\tau_{1}\right) / 2$

$$
\begin{aligned}
\hat{\varrho}_{\mathrm{e}}{ }^{0}(\tau)=\exp \left(-\frac{\mathrm{i}}{\hbar} \hat{\mathscr{F}}_{\mathrm{e}} \tau / 2\right) & \exp \left(\frac{\mathrm{i}}{\hbar} \hat{\mathscr{H}}_{\mathrm{g}} \tau / 2\right) \hat{\varrho}_{\mathrm{g}}{ }^{T} \times \\
& \exp \left(\frac{\mathrm{i}}{\hbar} \hat{\mathscr{F}}_{\mathrm{g}} \tau / 2\right) \exp \left(-\frac{\mathrm{i}}{\hbar} \hat{\mathscr{F}}_{\mathrm{e}} \tau / 2\right)
\end{aligned}
$$

and $\hat{\varrho}_{g}{ }^{T}$ is the density matrix of the ground equilibrium state with temperature $T$.

The quantities of interest are the averaged values of (i) the density matrix for calculation of the absorption spectrum and (ii) the coordinates and momenta as initial conditions for further MD simulation of the excited state dynamics. In the limits of the harmonic approximation, eq 9 determines a generalized Gaussian density matrix and, using standard techniques for calculation of averages, analytical expressions can be obtained for the quantities of interest. The absorption or $0^{0}$ dispersed fluorescence spectra $\left(0^{\circ}\right.$ denotes the vibrationless excited electronic state) of the molecule is calculated in the dipole and Condon approximation using the following formula for the transition probability: 47.52

$$
P\left(\omega_{\mathrm{L}}\right)=\left(\Omega_{\mathrm{L}}{ }^{2} / 4\right) \operatorname{Re} \int \mathrm{d} \tau f_{\mathrm{p}}(\tau) \exp \left[\mathrm{i}\left(\omega_{\mathrm{L}}-\omega_{\mathrm{ge}}\right) \tau\right] \chi(\tau)
$$

where $f_{\mathrm{p}}(\tau)=\int \mathrm{d} s u_{\mathrm{p}}(s-\tau / 2) u_{\mathrm{p}}{ }^{*}(s+\tau / 2)$ is the laser pulse autocorrelation function; $\Omega_{\mathrm{L}}=E_{\mathrm{L}} d_{\mathrm{ge}} / \hbar$ is the Rabi frequency; $\chi(\tau)$ is the linear response function ${ }^{53}$ given by ${ }^{47.52}$

$$
\begin{aligned}
& \chi(\tau)=\operatorname{Tr} \hat{\varrho}_{\mathrm{e}}^{0}(\tau)= \\
& \frac{\operatorname{det}\left[\sinh \left(\hbar \boldsymbol{\Omega}^{\prime \prime} / k T\right) /\left(\mathbf{s}_{1} \mathbf{s}_{2}\right)\right]}{\operatorname{det}^{1 / 2}\left(\mathbf{c}_{1} / \boldsymbol{\Omega}^{\prime \prime}+\mathbf{c}_{2} / \boldsymbol{\Omega}^{\prime}\right) \operatorname{det}^{1 / 2}\left(\mathbf{c}_{1} \mathbf{\Omega}^{\prime \prime}+\mathbf{c}_{2} \mathbf{\Omega}^{\prime}\right)} \times \\
& \exp \left[-\frac{1}{\hbar} \mathbf{D}^{\mathrm{T}} \cdot\left(\mathbf{c}_{\mathbf{1}} / \boldsymbol{\Omega}^{\prime \prime}+\mathbf{c}_{\mathbf{2}} / \boldsymbol{\Omega}^{\prime}\right)^{-1} \cdot \mathbf{D}\right] \\
& \mathbf{s}_{1}=\sinh \left[\mathbf{\Omega}^{\prime \prime}(\hbar / k T-\mathrm{i} \tau)\right], \quad \mathbf{s}_{\mathbf{2}}=\sinh \left(\mathrm{i} \boldsymbol{\Omega}^{\prime} \tau / 2\right) \\
& \mathbf{c}_{1}=\operatorname{coth}\left[\boldsymbol{\Omega}^{\prime \prime}(\hbar / k T-\mathrm{i} \tau) / 2\right], \quad \mathbf{c}_{2}=\operatorname{coth}\left(\mathrm{i} \boldsymbol{\Omega}^{\prime} \tau / 2\right)
\end{aligned}
$$

Here, when the Dushinsky rotation matrix is diagonal (eq 5), the displacement vector $\mathbf{D}$ is given simply by eq 6 . Equations 10 and 11 provide the quantum expression for the one-photon transition probability in terms of the ground and excited state frequency matrices, the displacements of the two surfaces, and the temperature, which is the complete set of parameters characterizing a harmonic molecule.

For the averaged normal coordinates after the excitation, we have

$$
\mathbf{Q}_{\mathrm{av}}^{\prime}=\mathbf{Q}_{0}^{\prime}+\int p(\tau) \Delta \mathbf{Q}(\tau) \mathrm{d} \tau / P\left(\omega_{\mathrm{L}}\right)
$$

where $\mathbf{Q}_{0}^{\prime}$ is the minimum energy configuration on the excited electronic state $|\mathrm{e}\rangle ; p(\tau)$ is the time-density expression, $\mathrm{d} P\left(\omega_{\mathrm{L}}\right) /$ $\mathrm{d} \tau$ (see eq 10 ); and $\Delta \mathbf{Q}(\tau)$ is the (dynamical) displacement vector corresponding to time interval $\tau$ and given by ${ }^{47,52}$

$$
\Delta \mathbf{Q}(\tau)=\frac{\operatorname{Tr} \Delta \hat{Q} \hat{\varrho}_{\mathrm{e}}^{0}(\tau)}{\operatorname{Tr} \hat{\varrho}_{\mathrm{e}}^{0}(\tau)}=-\mathbf{\Omega}^{-1} \mathbf{s}_{2}{ }^{-1}\left(\mathbf{c}_{1} / \mathbf{\Omega}^{\prime \prime}+\mathbf{c}_{2} / \mathbf{\Omega}^{\prime}\right)^{-1} \mathbf{D}
$$

The initial momentum distribution is not changed during the excitation because the Hamiltonians $\mathscr{T}_{\mathrm{g}}$ and $\mathscr{K}_{\mathrm{e}}$ are displaced from each other only in coordinate space and not in momentum space.

The results given above, eqs $10-13$, explore the advantages of time-dependent methods, in particular the density matrix technique, and avoid the necessity of calculating the entire spectrum of eigenvalues and eigenvectors. Furthermore, the 
use of Gaussian functions and operators allows the derivation of analytical closed from expressions for the quantities of interest. While the harmonic approximation has been used to obtain these results, more general results for spectral calculations that include anharmonicity, Dushinsky rotation, and dispersed fluorescence emission from any vibrational level in the excited state are also available due to Yan and Mukamel. ${ }^{49}$ The main innovation presented here is the use of the results for harmonic potentials to generate a method for creating quasiclassical initial conditions that simulate the nonstationary state prepared by an ultrashort pulse for a molecule at finite (nonzero) temperature.

The general procedure for computing the electronic spectra and generating quasiclassical initial conditions is as follows. First, the temperature is fixed and, for each normal mode, the displacement $D_{j}$ and frequencies $\left(\omega^{\prime \prime}{ }_{j}, \omega_{j}^{\prime}\right)$ on both the ground and excited states are specified. The function $\chi(\tau)$ is calculated using eq 11 for $N$ points (typically $N=8192$ ) with time increments $\Delta \tau=[(N-1) \Delta \omega]^{-1}$, where $\Delta \omega$ is the spectral resolution of the calculation. A standard FFT routine is used to obtain the absorption spectrum via eq 10 . Equation 13 is then used to sample the phase space of the wave packet on the excited state. It represents the quantum delocalization due to molecular dynamics during the laser pulse excitation, which supplements that due to the zero-temperature uncertainty spread and the Boltzmann thermal distribution in the ground electronic state. Next, the laser pulse width $\left(\tau_{\mathrm{L}}\right)$, the form of $u_{\mathrm{p}}(t)$, and the central frequency $\omega_{\mathrm{L}}$ are specified and the displacements $\Delta \mathbf{Q}(\tau)$ calculated from eq 13 for time intervals $\tau$ within the laser pulse duration. Each calculated vector $\Delta \mathbf{Q}$ is added to the equilibrium normal coordinate vector and transformed to Cartesian coordinates to obtain a set of initial conditions for a MD trajectory.

The number of initial conditions generated this way determines the size of the trajectory ensemble used to study the excited state dynamics. Thus, a sufficiently large number must be used to obtain statistically meaningful results; in this case, about 300 trajectories appears to be adequate. Finally, we note that eq 12 gives the averaged normal coordinates after the excitation (the center of the multidimensional wave packet) and could be used for calculating the initial conditions of a single representative MD trajectory in the case of very large system or limited computational resources.

Empirical Potential Energy Surfaces for $S_{0}$ and $S_{1}$. One of the primary goals of this work is to construct a global empirical potential surface for the ground and first excited states of stilbene using generalized force terms based on physical and chemical bonding considerations and which contain parameters that may be transferable. For this reason, we adopt the approach of the molecular mechanics method (MM3) ${ }^{54}$ which provides a force field formulated in terms of internal coordinates, that is, bond lengths, valence bond angles, dihedral torsional angles, and interatomic distances between nonbonded atoms. The MM potential is not a force field in the sense of a normal coordinate analysis, i.e., derived from the vibrational frequencies, but rather a collection of potential functions with transferable parameters, ${ }^{54-57}$ and most important for the present study, it can be used to explore large deviations from equilibrium structures. The parameters used in the present potential surfaces are derived from a number of sources: (i) computed fits of high-resolution spectroscopic data, including both line positions and relative intensities, available for trans-stilbene and 1,2-diphenylcyclobutene (an analog for cis-stilbene), (ii) reproduction of excited state decay lifetimes for cis- and trans-stilbene using classical dynamics simulations, (iii) structural data inferred from both experiment and electronic structure theory, and (iv) standard
MM3 parametrizations of vibrational degrees of freedom not directly probed by experiment.

A general rule in the development of the $\mathrm{MM}$ method is to use a minimum number of transferable parameters. Then, systematic errors in applications that remain after optimization will usually suggest places where further improvement of the force field may be desirable and the kind of modifications needed. The MM method was originally developed as a way to reproduce and predict molecular geometries; however, we impose the additional burden on the force field that it faithfully reproduce dynamical quantities. Since there is no systematic application of the method in studying intramolecular dynamics, we extend the philosophy of using the simplest parametrization possible by using the simplest possible forms for the potential functions that model the various force terms, especially for vibrations about which little is known. Also, when fitting torsional barrier parameters to excited state decay measurements, we examine dynamics that includes all vibrational degrees of freedom so as not to prejudice the outcome by using an overly constrained dynamical model. Finally, we also consider the additional effect of a bonding interaction between $C_{2}$ and $C_{2^{\prime}}$ (Figure 1) which allows for the flow of products into the DHP channel of the isomerization reaction. The resulting potential has the form

$$
U\left(\mathbf{r}_{1}, \ldots, \mathbf{r}_{N}\right)=U_{\mathrm{s}}+U_{\mathrm{b}}+U_{\mathrm{tor}}+U_{\mathrm{vw}}+U_{\mathrm{C}_{2} \mathrm{C}_{2}}
$$

where $U_{\mathrm{s}}, U_{\mathrm{b}}, U_{\mathrm{tor}}$, and $U_{\mathrm{vw}}$ are contributions due to bond stretches, valence angle bends, torsional (dihedral) angle twists, and nonbonded interatomic van der Waals interactions, respectively. $U_{\mathrm{C}_{2} \mathrm{C}_{2}}$, represents the bonding potential between $\mathrm{C}_{2}$ and $\mathrm{C}_{2}$.

For the valence bond stretch and bending forces, we assume harmonic oscillator potentials:

$$
\begin{gathered}
U_{\mathrm{s}}=0.5 \sum_{b} k_{b}\left(b-b_{0}\right)^{2} \\
U_{\mathrm{b}}=0.5 \sum_{\varphi} k_{\varphi}\left(\varphi-\varphi_{0}\right)^{2}
\end{gathered}
$$

in which $b_{0}$ and $\varphi_{0}$ are "equilibrium" values for the bond, $b$, and the angle, $\varphi$, respectively, and $k_{b}$ and $k_{\varphi}$ are the force constants. The equilibrium position parameters above represent "ideal" values for the local interaction-the actual equilibrium values are determined from the balance between these local potentials and the van der Waals and torsional interactions.

The potentials for the torsional angles $\theta$ are modeled by a truncated Fourier series,

$$
\begin{array}{r}
U_{\text {tor }}=0.5 \sum_{\theta}\left\{V_{2}[1-\cos (2 \theta)]+V_{4}[1-\cos (4 \theta)]\right\}= \\
\sum_{\theta}\left[V_{2} \sin ^{2}(\theta)+V_{4} \sin ^{2}(2 \theta)\right]
\end{array}
$$

a form which has been widely applied to large molecules with extended $\pi$-systems. Here for each different type of torsional angle in the molecule one must set the values of the force constants $V_{2}$ and $V_{4}$. For this functional form, $V_{2}$ determines the height of the barrier to rotation around a bond in the absence of steric interactions; in other words it provides a measure of the disruption of the $\pi$-system conjugation as rotation occurs about the bond. $V_{4}$ affects the shape of the potential as shown in Figure 2. If $V_{2}>0$, which is typical for the ground state of a conjugated molecule (i.e., minima occur at planar configurations), the barrier is at $90^{\circ}$ and the region of the minimum ( $\theta$ 
$=0^{\circ}$ ) becomes flatter as $V_{4}$ decreases (Figure 2b). If $V_{2}<0$ there is a minimum at $90^{\circ}$ and the maxima become flatter as $V_{4}$ increases. When $V_{4}>1 / 4\left|V_{2}\right|$, the maxima become local minima separated from the global minimum by a small barrier near $\theta=0^{\circ}$ and this barrier gets larger and moves to larger $\theta$ as $V_{4}$ increases further (see Figure $2 a$ ).

Van der Waals (nonbonded) interactions are calculated for all pairs of atoms $(i, j)$ which do not belong to the same chemical bond or the same valence angle using a Hill-type potential ${ }^{58}$ involving two parameters for each atom; one is the van der Waals radius of the atom, and the other is an energy scale factor. The function representing the potential consists of an attractive term varying as $r^{-6}$, which represents the dipole/induced dipole interaction, and a repulsive term which is exponential. A latent problem with this type of function is that at small interatomic distances the potential turns over and becomes strongly attractive. To prevent this from becoming a factor in the present study, an exponential repulsion term is fit to the potential function at the inflection point so that the potential and its first derivative are continuous

$U_{\mathrm{vW}}=$

$\sum_{r} \begin{cases}f\left[-2.25 / r_{n}{ }^{6}+8.28 \times 10^{5} \exp \left(-r_{n} / 0.0736\right)\right], & \text { if } r \geq r_{0} \\ V \exp \left(-k r_{n}\right), & \text { otherwise }\end{cases}$

Here $f$ is proportional to the depth of the potential and depends on the identity of the two atoms; $r_{n}=r_{i j} / s$, where $s$ is sum of the van der Walls radii for atoms $i$ and $j$, and $r_{i j}=\left|\mathbf{r}_{i}-\mathbf{r}_{j}\right|$.

The bonding interaction between $\mathrm{C}_{2}$ and $\mathrm{C}_{2}$ presents a major challenge. Since little is known about the shape of the PES along this coordinate, a Morse function is used

$$
\begin{aligned}
U_{\mathrm{C}_{2} \mathrm{C}_{2^{\prime}}}=D_{\mathrm{CC}}\left\{\operatorname { e x p } \left[-2 \beta_{\mathrm{c}}\left(R_{\mathrm{CC}}-\right.\right.\right. & \left.\left.R_{\mathrm{eq}}\right)\right]- \\
& \left.2 \exp \left[-\beta_{\mathrm{c}}\left(R_{\mathrm{CC}}-R_{\text {eq }}\right)\right]\right\}
\end{aligned}
$$

where $D_{\mathrm{CC}}$ is the bond dissociation energy, $\beta_{\mathrm{c}}$ determines the range of the interaction and (with $D_{\mathrm{CC}}$ ) the vibrational frequency of the bond in DHP, $R_{\mathrm{eq}}$ is the "ideal" equilibrium bond length as discussed earlier, and $R_{\mathrm{CC}}$ is the actual distance between $\mathrm{C}_{2}$ and $\mathrm{C}_{2^{\prime}}$.

As a standard molecular modeling procedure, one specifies an initial guess for the equilibrium configuration of the molecule. This could, for example, be the geometry implied by the idealized equilibrium coordinate parameters in the force field. Then, through minimization of the potential energy ${ }^{54}$ the equilibrium geometry is found. After mass-weighting the Cartesian coordinates, the force constant matrix is diagonalized giving the squared normal mode frequencies (eigenvalues) and the unitary (mass-weighted Cartesian to normal coordinates) transformation matrix, $V$, whose columns are the eigenvectors corresponding to each normal mode. By starting from different initial configurations on the PES, this procedure can lead to different local minima, corresponding to different conformers, but these are easily distinguished and characterized for molecules the size of stilbene. The parameters of the force field are then adjusted until a good fit to the geometry, energy separation between conformers, and frequencies, as known from spectroscopic experiments or $a b$ initio calculations, is obtained.

We note that in the specified empirical force field, eqs 1419 , the anharmonic and coupling elements in the normal mode representation are determined not only from the force terms represented by general functional forms, eqs 17 and 18 , but also from the quadratic forms in eqs 15 and 16 due to the nonlinear transformation from internal to Cartesian coordinates. In fact, the most significant contribution to the third- and fourth-order potential energy derivatives is due to the bond stretching potential in eq 15. This issue has been discussed extensively by Sibert and co-workers ${ }^{59}$ in the context of comparing power series expansions of potential surfaces in Cartesian versus internal coordinate normal modes. In the present work, little $a$ priori information beyond the harmonic approximation is available; however, in general the dynamics explored by the system remains in the harmonic regime for most of the vibrational degrees of freedom.

While the procedure outlined above is well established for ground state potentials, the construction of the excited electronic state surface is more difficult. In our model, we use the same mathematical forms for terms in the force field, eqs 14-19, as for the ground state. This is a rough approximation; however, an appropriate use of the potential parameters allows us to represent major characteristics of the surface and to probe different models. For instance, the ethylenic torsional potential modeled by eq 17 is flexible enough to allow for inverted ground and excited states, where the maximum at $90^{\circ}$ in the ground state corresponds to a minimum in the excited state (see Figure 2). Moreover, a straightforward modification of the parameters $V_{2}$ and $V_{4}$ allows one to place a local barrier near the $0^{\circ}$ position (corresponding to excited state cis-stilbene) with different heights and position (see Figure 2a). ${ }^{47}$ In addition, the use of a Morse potential, eq 19 , for chemical bonds generally permits the study of both bound and dissociative dynamics. In the present work, the Morse potential form is used only for degrees of freedom associated with a reaction coordinate (such as $R_{\mathrm{C}_{2} \mathrm{C}_{2}}$ ). Otherwise, a simple harmonic potential is used to treat bond stretches because, at the energies considered, most bond displacements are small compared to the equilibrium bond lengths during the course of the dynamics.

Using this empirical approach, we attempt to utilize as much experimental information as is available to optimize the force field parameters and qualitatively determine the shape of the excited state surface. In contrast with the construction of the ground state surface, we divide the modeling of the excited state potential into two parts: (i) the Franck-Condon region for both the cis and trans isomers is optimized by simulating the appropriate observed electronic absorption spectra and (ii) the torsional barrier potentials for cis-trans isomerization and the nonadiabatic coupling to the ground state are fit by examining the excited state dynamics and reproducing decay lifetimes.

As a starting point for the spectrum-fitting procedure, we have the ground state potential surface which specifies the equilibrium structure, the vibrational frequencies, and the identity of the ground state normal modes in terms of individual atom motions. Although the normal modes are mixtures of different internal coordinates, some of them may be distinguished as having mostly stretching, or angle deformation, or torsional character. To simplify our spectral fit, we assume that there is no Dushinsky rotation - that is, the normal modes have the same composition on both the ground and excited states. Given that the excitation of the $\pi$-system is likely to cause a strong mixing of the normal modes involving large $\mathrm{C}-\mathrm{C}$ bond stretch components, this approximation may appear unjustified; however, most of the motions critical to stilbene isomerization reactions are low-frequency vibrations that likely do not mix strongly with the much higher frequency bond stretch modes. Any attempt to model the Dushinsky rotation of the stretch modes would be largely guesswork and unlikely to produce a potential surface that is any better justified than the one resulting from the present assumptions. As we discuss below in section 


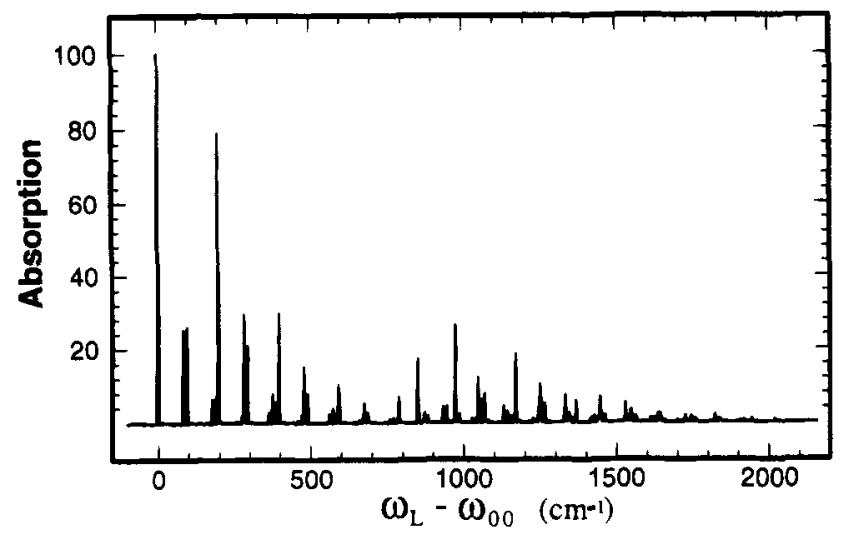

Figure 3. Calculated absorption spectrum of trans-stilbene. The calculation is made with $0.5 \mathrm{~cm}^{-1}$ resolution using eqs 10 and 11,14 active modes with frequencies and displacements given in Table 1, and a temperature of $5 \mathrm{~K}$. Relative intensities are used for evaluation of the displacements necessary to match the calculated and experimental ${ }^{34}$ spectra.

$\mathrm{V}$, the main effect of these modes is to provide a vibrational "bath" for the dynamics which gradually dissipates energy from the primary isomerization coordinates.

By adjusting the corresponding force constants and equilibrium parameters in eqs $14-19$, one can generally reproduce the desired frequency and intensity of the corresponding line in the absorption spectrum. This procedure is iterative and includes specifying the parameters for the excited state, then calculating the vibrational frequencies and the vector of displacements, and finally comparing the simulated spectrum with experimental data. A time-saving sophistication is to first determine the characteristics of the prominent modes in the spectrum. The experimental spectrum ${ }^{34-37}$ provides the frequencies $\left(\omega^{\prime \prime}{ }_{j}, \omega_{j}^{\prime}\right)$ of the prominent modes in the spectrum and their relative intensities. From these we can iteratively determine the vector of displacements $\left(D_{j}\right)$. This simulation is straightforward as the modes are taken to be independent of one another and a few calculations are sufficient to obtain excellent correspondence with the experimental spectrum, limited only by the validity of the constraints imposed by the model, i.e, the neglect of anharmonicities and Dushinsky rotation.

Figure 3 shows the calculated absorption spectrum, at a resolution of $0.5 \mathrm{~cm}^{-1}$, using eqs 10 and 11 and the frequencies and displacements for the 14 normal modes listed in Table 1. The frequencies are taken from the high-resolution supersonic jet results ${ }^{34}$ and assignments given in Tables $1-3$ or ref 34 . We note that Chiang and Laane ${ }^{60}$ have recently published a revised interpretation of the trans-stilbene electronic absorption spectrum, and we are presently investigating the sensitivity of our dynamical results in light of their revisions. The displacements are calculated as given above, and the calculated spectrum compares favorably with the experimental spectrum given in Figure 4 of ref $34 .{ }^{47}$ The excited state vibrational frequency and dimensionless normal mode displacement data in Table 1 are then fit by adjusting the excited state MM parameters, thus avoiding further calculation of the spectrum. The iterative procedure is reduced to specifying the parameters for the excited state, calculation of the frequencies and displacements, and comparison with the already fixed ones.

Despite the simplifying assumptions that are made, the spectral simulation is not straightforward as the modes are mixed and changing the parameters connected with an internal coordinate will lead to changes in a number of modes. Consequently, a large number of iterations is necessary and only
TABLE 1: Vibrational Frequencies $\left(\omega^{\prime \prime}, \omega^{\prime}\right)$, Dimensionless Displacements $(\bar{D})$ of 14 Optically Active Modes of trans-Stilbene Used in the Calculation of the Absorption Spectrum of Figure 3, and the Intensities of the Fundamentals in the Calculated and Experimental (in Parentheses) Spectra ${ }^{a}$

\begin{tabular}{ccccc}
\hline$\omega^{\prime \prime}, \mathrm{cm}^{-1}$ & $\omega^{\prime}, \mathrm{cm}^{-1}$ & $\bar{D}$ & $I\left(I_{\text {exp }}\right), \mathrm{au}^{b}$ & mode $^{c}$ \\
\hline 75.0 & 95.1 & 0.73 & $26.0(26)$ & $\nu 37$ \\
119.0 & 83.2 & 0.72 & $25.1(25)$ & $\nu 36$ \\
204.0 & 197.6 & 1.27 & $78.8(79)$ & $\nu 25$ \\
290.0 & 280.3 & 0.46 & $29.4(30)$ & $\nu 24$ \\
624.0 & 590.7 & 0.44 & $10.1(10)$ & $\nu 23$ \\
870.0 & 850.0 & 0.66 & $17.2(17)$ & $\nu 21$ \\
1006.0 & 972.7 & 0.83 & $26.4(26)$ & $\nu 20$ \\
1031.0 & 1249.3 & 0.58 & $10.3(10.6)$ & $\nu 18$ \\
1072.0 & 1069.8 & 0.26 & $5.8(5)$ & $\nu 13$ \\
1340.0 & 1332.4 & 0.41 & $7.4(7.5)$ & $\nu 12$ \\
1495.0 & 1464.0 & 0.14 & $1.9(1.7)$ & $\nu 10$ \\
1584.0 & 1548.4 & 0.38 & $3.4(3.5)$ & $\nu 9$ \\
1607.0 & 1553.0 & 0.29 & $1.8(1.9)$ & $\nu 8$ \\
1654.0 & 1637.8 & 0.26 & $2.3(2.3)$ & $\nu 7$
\end{tabular}

a The frequencies and experimental intensities are from ref 34 ; the displacements are calculated iteratively for a good correspondence between the calculated and experimental intensities of the fundamentals. ${ }^{b}$ The $0-0$ line is with intensity equal to $100 .{ }^{c}$ Assignments as in ref 34.

limited precision can be attained given the simple form of the potential functions. Nevertheless, even if an exact determination of the MM parameters is not possible, their values can be confined to a fairly narrow range because the relative intensities are very sensitive to changes in these parameters. For example, changes in the bond length parameters, $b_{0}$, of only $0.01 \AA$ lead to a qualitatively different relative intensity pattern from that seen in the experimental spectrum. As a result, we have carefully determined optimized parameters for the potential but also checked to ensure that small changes in these parameters do not affect substantially the results from the dynamical calculations. Table 2 lists the calculated MM parameters for both the ground and excited states.

With these parameters the agreement of the calculated spectrum with the experimental is worse than for the spectrum calculated with the optimal displacements (Figure 3 and Table 1). For 10 of the active modes the intensities are within $20 \%$ deviation from the experimental, and for the other 4 modes within $35 \%$. At the same time, if any of the modes is considered separately, an agreement within $10 \%$ of the experimental intensities could be obtained by additionally changing some of the parameters related to the particular mode. These changes determine different (from Table 2) sets of parameters for the excited state ("check-parameters"). All of the results given below are obtained with the parameters from Table 2; however, additional calculations with the "check-parameters" give the same qualitative results and small quantitative changes.

Using the parameters from Table 2 in the molecular force field (eqs 14-19), the cis-stilbene equilibrium geometry in Cartesian coordinates is obtained from trans-stilbene by a rotation of $180^{\circ}$ about the ethylenic bond, followed by a minimization of the energy. We note that the torsional potential functions are designed to favor a planar geometry. This is realized in trans-stilbene, but in cis-stilbene, the balance between these forces and the steric interactions between the two phenyl rings results in an optimized structure with an ethylenic torsional angle of $\theta=13.6^{\circ}$, phenyl twist angles of $\phi_{1}=34.2^{\circ}$ and $\phi_{2}$ $=36.3^{\circ}$, and central valence bond angles of $\alpha_{1}=129.8^{\circ}$ and $\alpha_{2}=129.3^{\circ}$, in the excited state. Although the excited state equilibrium geometry is not known experimentally, comparison with the experimental values ${ }^{48}$ of $\phi_{2}=45 \pm 10^{\circ}$ and $\alpha_{1}=$ $129.5^{\circ}$, and the theoretically predicted value $\theta=9^{\circ} 12$ for the 
TABLE 2: Parameters for the MM PESs Used in MD Simulations of Stilbene Isomerization Dynamics

(a) Parameters for Bond Stretching Potentials (Eq 15)

\begin{tabular}{lccccc}
\hline & \multicolumn{2}{c}{ ground state } & & \multicolumn{2}{c}{ excited state } \\
\cline { 2 - 3 } \cline { 5 - 6 } \multicolumn{1}{c}{ bond } & $\begin{array}{c}k_{\mathrm{b}}, \mathrm{kcal} / \\
\left(\mathrm{mol} \AA^{2}\right)\end{array}$ & $b_{0}, \AA$ & & $\begin{array}{l}k_{\mathrm{b}}^{\prime}, \mathrm{kcal} / \\
\left(\mathrm{mol} \AA^{2}\right)\end{array}$ & $b_{0}^{\prime}, \AA$ \\
\hline $\mathrm{C}_{\mathrm{ph}}-\mathrm{C}_{\mathrm{ph}}$ & 993.7 & 1.397 & & 893.0 & 1.397 \\
$\mathrm{C}_{\mathrm{ph}}-\mathrm{H}$ & 725.6 & 1.113 & & 725.0 & 1.113 \\
$\mathrm{C}_{\mathrm{e}}-\mathrm{C}_{\mathrm{e}}$ & 1150.0 & 1.338 & & 750.0 & 1.358 \\
$\mathrm{C}_{\mathrm{ph}}-\mathrm{C}_{\mathrm{e}}$ & 818.0 & 1.458 & & 1100.0 & 1.435 \\
$\mathrm{C}_{\mathrm{e}}-\mathrm{H}$ & 725.6 & 1.113 & & 720.0 & 1.114
\end{tabular}

(b) Parameters for Angle Deformation Potentials (Eq 16)

\begin{tabular}{|c|c|c|c|c|}
\hline \multirow[b]{2}{*}{ angle } & \multicolumn{2}{|c|}{ ground state } & \multicolumn{2}{|c|}{ excited state } \\
\hline & $\begin{array}{c}k_{4}, \mathrm{kcal} / \\
\left.(\mathrm{mol} \mathrm{deg})^{2}\right)\end{array}$ & $\begin{array}{l}\varphi_{0} \\
\text { deg }\end{array}$ & $\begin{array}{c}k_{4}^{\prime}, \mathrm{kcal} / \\
\left(\mathrm{mol} \mathrm{deg}^{2}\right)\end{array}$ & $\begin{array}{l}\varphi_{0}^{\prime} \\
\text { deg }\end{array}$ \\
\hline $\mathrm{C}_{\mathrm{ph}}-\mathrm{C}_{\mathrm{ph}}-\mathrm{C}_{\mathrm{ph}}$ & 0.0016 & 120 & 0.0013 & 120.1 \\
\hline $\mathrm{C}_{\mathrm{ph}}-\mathrm{C}_{\mathrm{ph}}-\mathrm{H}$ & 0.0014 & 120 & 0.0011 & 120.4 \\
\hline $\mathrm{C}_{\mathrm{ph}}-\mathrm{C}_{\mathrm{ph}}-\mathrm{C}_{\mathrm{e}}$ & 0.0010 & 120 & 0.0008 & 121.3 \\
\hline $\mathrm{C}_{\mathrm{ph}}-\mathrm{C}_{\mathrm{e}}-\mathrm{H}$ & 0.0012 & 120 & 0.0010 & 120.0 \\
\hline $\mathrm{C}_{\mathrm{ph}}-\mathrm{C}_{\mathrm{e}}-\mathrm{C}_{\mathrm{z}}$ & 0.0014 & 120 & 0.0008 & 121.7 \\
\hline $\mathrm{C}_{\mathrm{e}}-\mathrm{C}_{\mathrm{e}}-\mathrm{H}$ & 0.0015 & 120 & 0.0009 & 120.8 \\
\hline
\end{tabular}

(c) Parameters for Torsional Potentials (Eq 17)

\begin{tabular}{lccccc}
\hline & \multicolumn{2}{c}{ ground state } & & \multicolumn{2}{c}{ excited state } \\
\cline { 2 - 3 } \multicolumn{1}{c}{ atoms } & $\begin{array}{c}V_{2}, \mathrm{kcal} / \\
\mathrm{mol}\end{array}$ & $\begin{array}{c}V_{4}, \\
\mathrm{kcal} / \mathrm{mol}\end{array}$ & & $\begin{array}{c}V_{2}^{\prime}, \mathrm{kcal} / \\
\mathrm{mol}\end{array}$ & $\begin{array}{c}V_{4}^{\prime} \\
\mathrm{kcal} / \mathrm{mol}\end{array}$ \\
\hline $\mathrm{C}_{\mathrm{ph}}-\mathrm{C}_{\mathrm{ph}}$ & 3.00 & 0 & & 2.95 & 0 \\
$\mathrm{C}_{\mathrm{ph}}-\mathrm{C}_{\mathrm{ph}}-\mathrm{C}_{\mathrm{e}}-\mathrm{C}_{\mathrm{e}}$ & 6.245 & 0 & & 6.25 & -1.72 \\
$\mathrm{C}_{\mathrm{ph}}-\mathrm{C}_{\mathrm{ph}}-\mathrm{C}_{\mathrm{e}}-\mathrm{H}$ & 3.00 & 0 & & 3.00 & 0 \\
$\mathrm{C}_{\mathrm{ph}}-\mathrm{C}_{\mathrm{e}}-\mathrm{C}_{\mathrm{e}}-\mathrm{C}_{\mathrm{ph}}$ & 29.00 & 0 & & -32.90 & 11.58 \\
$\mathrm{C}_{\mathrm{ph}}-\mathrm{C}_{\mathrm{e}}-\mathrm{C}_{\mathrm{e}}-\mathrm{H}$ & 3.00 & 0 & & 2.95 & 0 \\
$\mathrm{H}-\mathrm{C}_{\mathrm{e}}-\mathrm{C}_{\mathrm{e}}-\mathrm{H}$ & 3.00 & 0 & & 2.95 & 0
\end{tabular}

(d) Parameters for van der Waals Potentials (Eq 18)

\begin{tabular}{lcccc}
\hline atom $(\mathrm{s})$ & $f, \mathrm{kcal} / \mathrm{mol}$ & $r_{\mathrm{vdw}}, \AA$ & $V, \mathrm{kcal} / \mathrm{mol}$ & $k, \AA^{-1}$ \\
\hline $\mathrm{C}-\mathrm{C}$ & 0.107 & & & \\
$\mathrm{C}-\mathrm{H}$ & 0.067 & & 42107.2 & 4.590 \\
$\mathrm{H}-\mathrm{H}$ & 0.042 & & 32351.0 & 5.741 \\
$\mathrm{C}$ & & 1.7 & & \\
$\mathrm{H}$ & & 1.2 & &
\end{tabular}

(e) Parameters for the Bonding Potential (Eq 19)

\begin{tabular}{|c|c|c|c|c|c|}
\hline \multicolumn{3}{|c|}{ ground state } & \multicolumn{3}{|c|}{ excited state } \\
\hline $\begin{array}{c}D_{\mathrm{CC}} \\
\mathrm{kcal} / \mathrm{mol}\end{array}$ & $\beta_{c}, \AA^{-1}$ & $R_{0}, \AA$ & $\begin{array}{c}D_{\mathrm{CC}}^{\prime}, \\
\mathrm{kcal} / \mathrm{mol}\end{array}$ & $\beta_{c}^{\prime}, \AA^{-1}$ & $R_{0}^{\prime}, \AA$ \\
\hline 35.0 & 4.67 & 1.53 & 39.0 & 2.00 & 1.82 \\
\hline
\end{tabular}

ground state of cis-stilbene indicates that the present surface is consistent with reasonable expectations for the excited state, especially given the simplicity of the PES. Finally, the potential along the cis-trans isomerization coordinate (ethylenic torsion), $\theta$, is modeled to give an appropriate energy gap between the ground and excited state surfaces at $\theta=90^{\circ}$ and to reproduce the excited state lifetime in dynamical simulations (see section IV below).

Although based on measurements that probe only a small portion of the excited state potential, the empirical excited state PES formed in this manner can be extrapolated to the whole conformation space using functional forms that represent the generalized forces in the molecule. This surface is shown as contour plots for various cross sections in the multidimensional surface in Figure 4. In stilbene the most important internal coordinates for the initial short-time excited state dynamics occur in the central part of the molecule along the ethylenic bridge, including the torsional $(\theta)$ and phenyl twist $(\phi)$ dihedral angles (see Figure $4 \mathrm{a}$ ), the central valence bending angles $(\alpha)$ (Figure $4 \mathrm{~b}$ ), and the internuclear distance between the $\mathrm{C}_{2}$ and $\mathrm{C}_{2}$ atoms
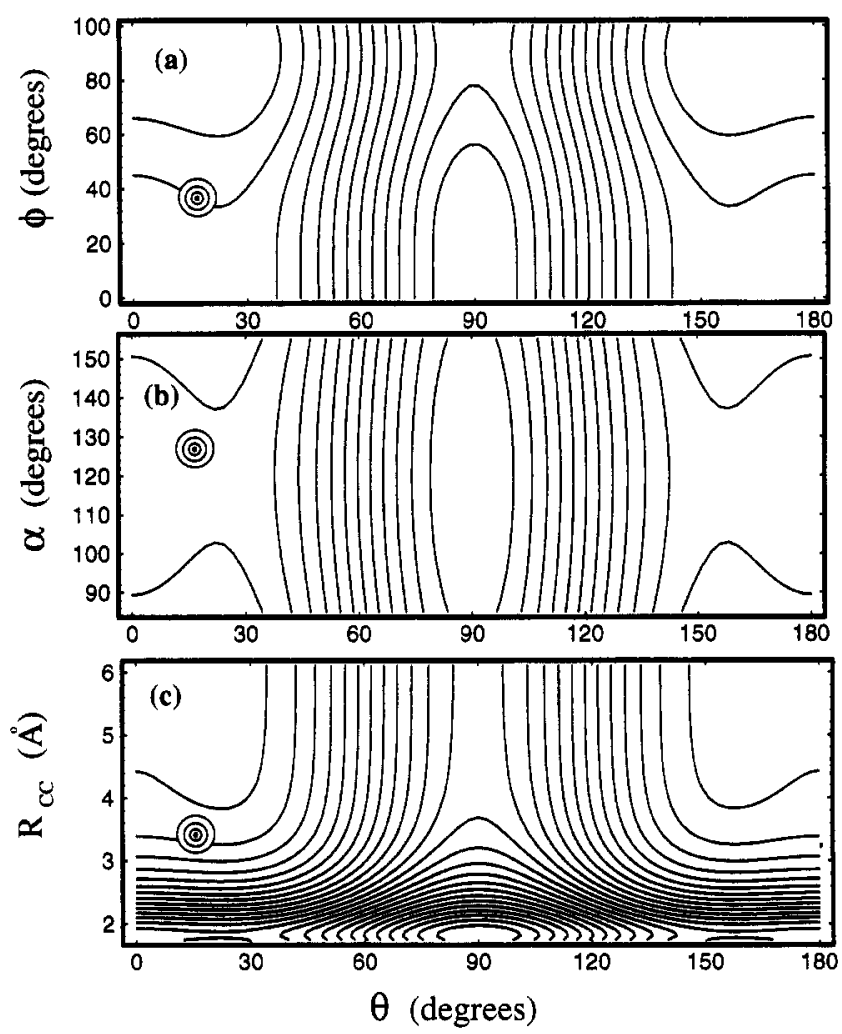

Figure 4. Potential energy surface for the $S_{1}$ state of stilbene: cross sections along the ethylenic torsional angle $(\theta)$ and (a) the phenyl twisting angle $(\phi)$, (b) the bending angle $(\alpha)$, and (c) the interatomic $\mathrm{C}_{2}-\mathrm{C}_{2}$ distance $\left(R_{\mathrm{CC}}\right)$. All other coordinates are fixed at their equilibrium values, and van der Waals interactions are not calculated. The absolute minima are at $\theta=90^{\circ}$ and $\phi=0^{\circ}$ (a), $\alpha=120^{\circ}$ (b), and $R_{\mathrm{CC}}=1.82 \AA$ (c); the contours are spaced evenly by $1000 \mathrm{~cm}^{-1}$. The smaller set of contours indicates the position of the Franck-Condon region in each case.

(Figure 4c). The plots in Figure 4 span both the trans and cis regions of the potential and are all symmetrical in the torsion angle $\theta$. The asymmetry of the overall potential surface, which is manifested in the well-known differences in the isomerization dynamics of the two isomers, can be attributed to steric effects which are much stronger in the cis than in the trans isomer. This was partly confirmed by earlier work in our group ${ }^{47}$ where the simulated excited state decay time for cis-stilbene is 295 fs, but for trans-stilbene, the decay time is $33.1 \mathrm{ps}$ on the same surface (the experimental value is $80 \mathrm{ps)}{ }^{61}$ From Figure 4 it is clear that the molecule is more flexible in the twist and valence angles and the photocyclization bond-forming coordinate, than in the torsional coordinate. This fact, combined with the mixed character of most of the optically active modes (see Table 2 of ref 43), suggests that the isomerization dynamics is likely to be a complex movement involving several of the important coordinates, rather than a direct motion along $\theta$.

\section{Excited State Dynamics}

The excited state dynamics of isolated cis-stilbene is modeled using an ensemble of classical trajectories. The initial conditions for the trajectory calculations, however, are chosen to accurately reflect the quantum state prepared by the excitation of a vibrationally cold harmonic molecule using an ultrashort laser pulse. Using the empirical PES for the ground and excited states, the frequency matrices and displacements are calculated for the 72 normal modes. Next, excitation with a $100 \mathrm{fs}$ Gaussian laser pulse with $4000 \mathrm{~cm}^{-1}$ excess vibrational energy is modeled using eqs 12 and 13 for the normal coordinates which 

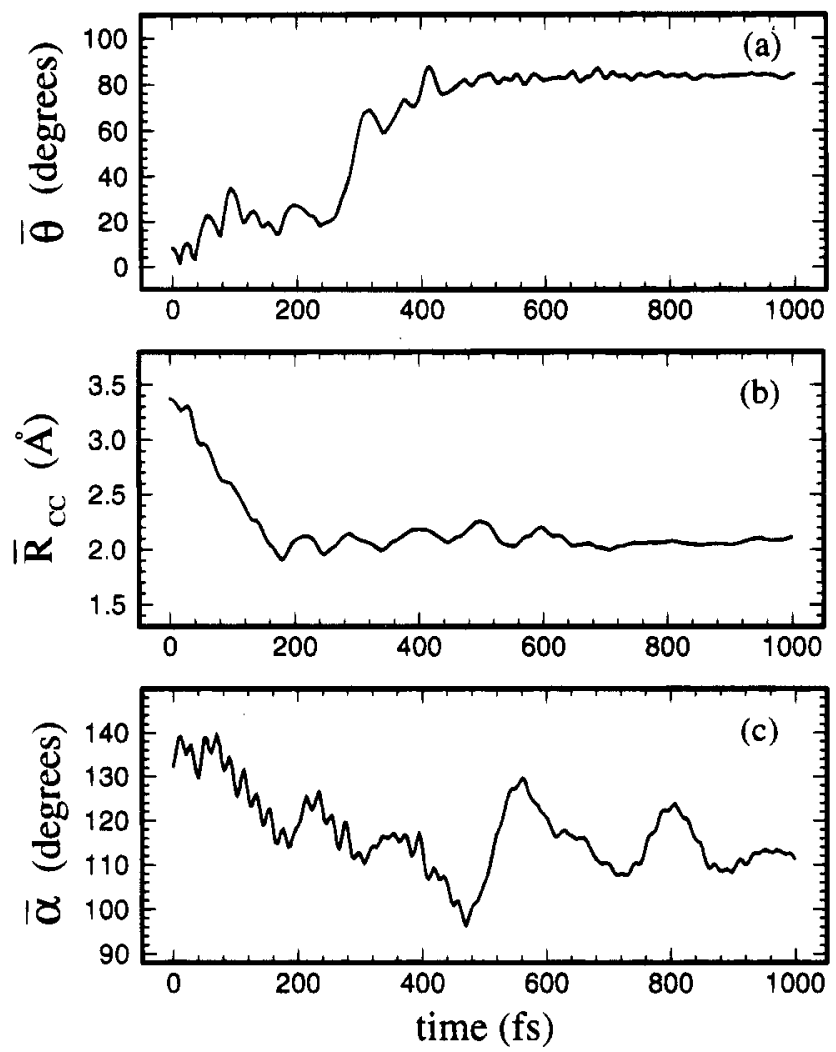

Figure 5. Excited state dynamics: averaged time dependence of (a) the ethylenic torsional angle $(\theta)$, (b) the interatomic $C_{2}-C_{2^{\prime}}$ distance, and (c) the bending angle $(\alpha)$. An ensemble of 300 trajectories is used for averaging, with initial conditions representing excitation with a 100 fs Gaussian laser pulse and $4000 \mathrm{~cm}^{-1}$ excess vibrational energy.

are then transformed to Cartesian coordinates. Initial conditions are generated for 300 molecular dynamics (MD) trajectories: the first set (eq 12) represents the averaged values over the laser pulse and can be thought of as the center of a multidimensional wave packet on the excited state surface; all others are generated (eq 13) with random values of $\tau$ within a $0-100$ fs time interval and represent the delocalization of the wave packet.

Several general observations can be made regarding the dynamics of the trajectory ensemble generated by these initial conditions. First, the average lifetime of the ensemble is 280 fs. This lifetime is arbitrarily defined as the time required for trajectories to escape from the Franck-Condon region by overcoming the local barrier $V_{\theta}$ along the ethylenic torsion coordinate (see Figure 2a). In spectroscopic experiments (pump-probe transient absorption or fluorescence decay) this represents the time for the system to leave the observation window. Its value depends mainly on the local barrier $V_{\theta}$, and the potential parameters were adjusted to reproduce approximately the experimental value of $307 \mathrm{fs} .{ }^{10}$ The cross-sectional barrier for torsion that best reproduces the experimental lifetime is $V_{\theta}=340 \mathrm{~cm}^{-1}$ and occurs at $\theta_{\mathrm{b}}=22.5^{\circ}$, but as we discuss later (section $\mathrm{V}$ ), its value is sensitive to the parameters of the twist torsional potential ${ }^{47}$ and a more conservative estimate is a value between 260 and $400 \mathrm{~cm}^{-1}$.

In Figure 5, the averaged time dependencies of the torsional angle $(\theta)$, the distance between the $C_{2}$ and $C_{2^{\prime}}$ atoms, and the bend angle $(\alpha)$ are shown. For the individual trajectories, typical characteristics include initial movement along the photocyclization $\mathrm{C}_{2}-\mathrm{C}_{2}$, coordinate from 3.4 to $\approx 2.0 \AA$ in $200-300 \mathrm{fs}$, as well as oscillations along the ethylenic torsion coordinate around $\theta=0^{\circ}$ with an amplitude of $8-20^{\circ}$. Most of the trajectories display a combination of these motions with torsional oscillation persisting until a time $t_{\theta}$ when the trajectory is able to overcome
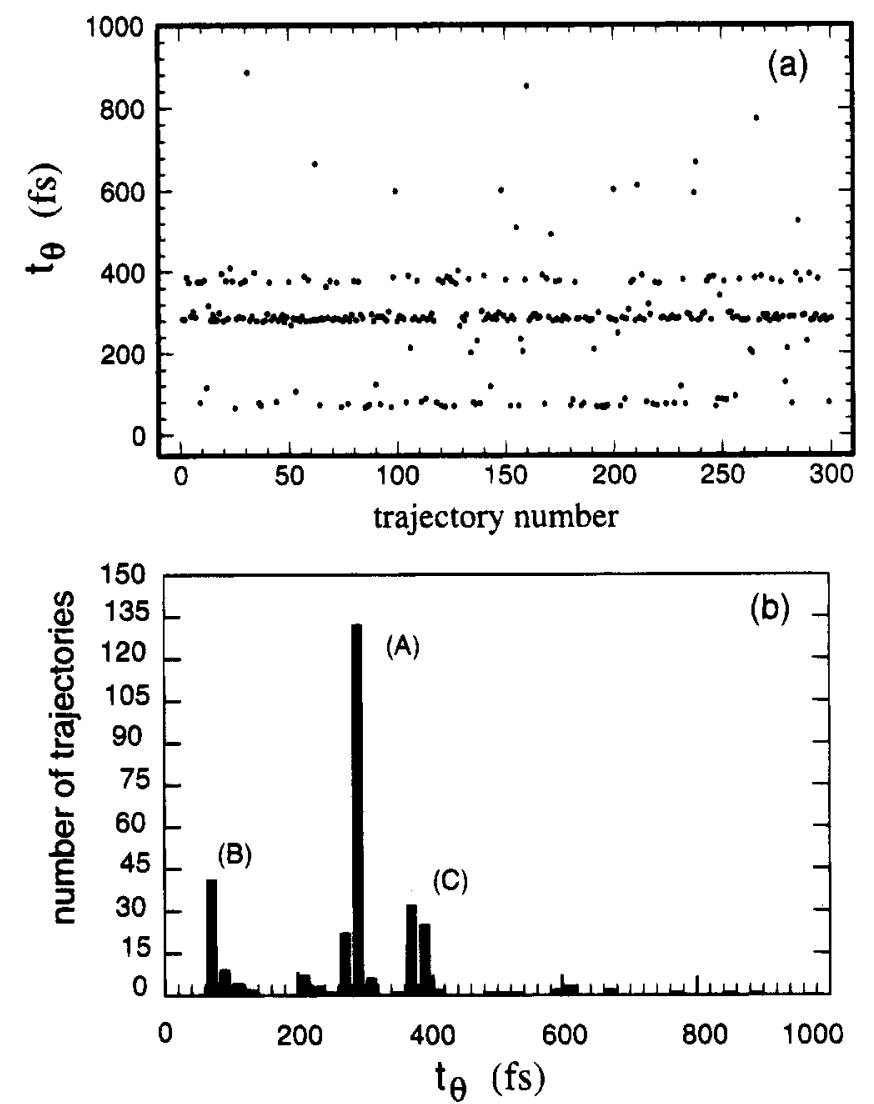

Figure 6. (a) Distribution of the decay time, $t_{\theta}$, in the ensemble of trajectories, where $t_{\theta}$ is determined as the time for leaving the FrankCondon region by overcoming the local barrier to isomerization; (b) column graph of (a): the columns represent the number of trajectories with $t_{\theta}$ in the corresponding $20 \mathrm{fs}$ interval.

the local barrier to cis-trans isomerization. Once the system passes over this barrier, it rapidly moves within 30 fs toward the excited state potential minimum at $\theta=90^{\circ}$ forming a vibrationally hot, twisted intermediate. The remainder of the vibrational modes do not appear to contribute significantly to the excited state dynamics, remaining predominantly lowamplitude vibrations; however, they participate in the dynamics by providing an energy bath in which the large amplitude motions take place.

Although the potential parameters are fit so that the average lifetime over the ensemble matches the lifetime observed experimentally, the distribution of the barrier crossing times, $t_{\theta}$, is not statistical (see Figure 6). For almost half of the trajectories (145), $t_{\theta}$ lies between 260 and $300 \mathrm{fs}$, close to the average value. However, two other groups of trajectories display markedly different lifetimes: 50 trajectories have $t_{\theta}$ between 60 and $100 \mathrm{fs}$, while 57 have $t_{\theta}$ between 360 and 400 fs. Consequently, the average time dependence of $\theta(t)$ (Figure 5a) shows small oscillations around $\theta=0^{\circ}$ in the first $60 \mathrm{fs}$, followed by a peak at $t \approx 80 \mathrm{fs}$ with oscillations around $\theta \approx$ $20^{\circ}$, and finally a transition to the $90^{\circ}$ region at $t \approx 280 \mathrm{fs}$. If this coordinate could be monitored experimentally, it would appear to indicate a minimum on the PES at $\theta \approx 20^{\circ}$ to which a transition is made in $\approx 80 \mathrm{fs}$. However, the present simulations indicate that this is actually a result of averaging over trajectories which decay on different time scales. In particular, this behavior can be largely attributed to the group of fast trajectories with $t_{\theta}$ between 60 and $100 \mathrm{fs}$. The actual local minimum for the torsional potential is near $\theta=0^{\circ}$.

A more detailed examination of the dynamics reveals that the three isomerization time scales correspond to three distinct pathways followed by the dynamics on the excited state surface. 


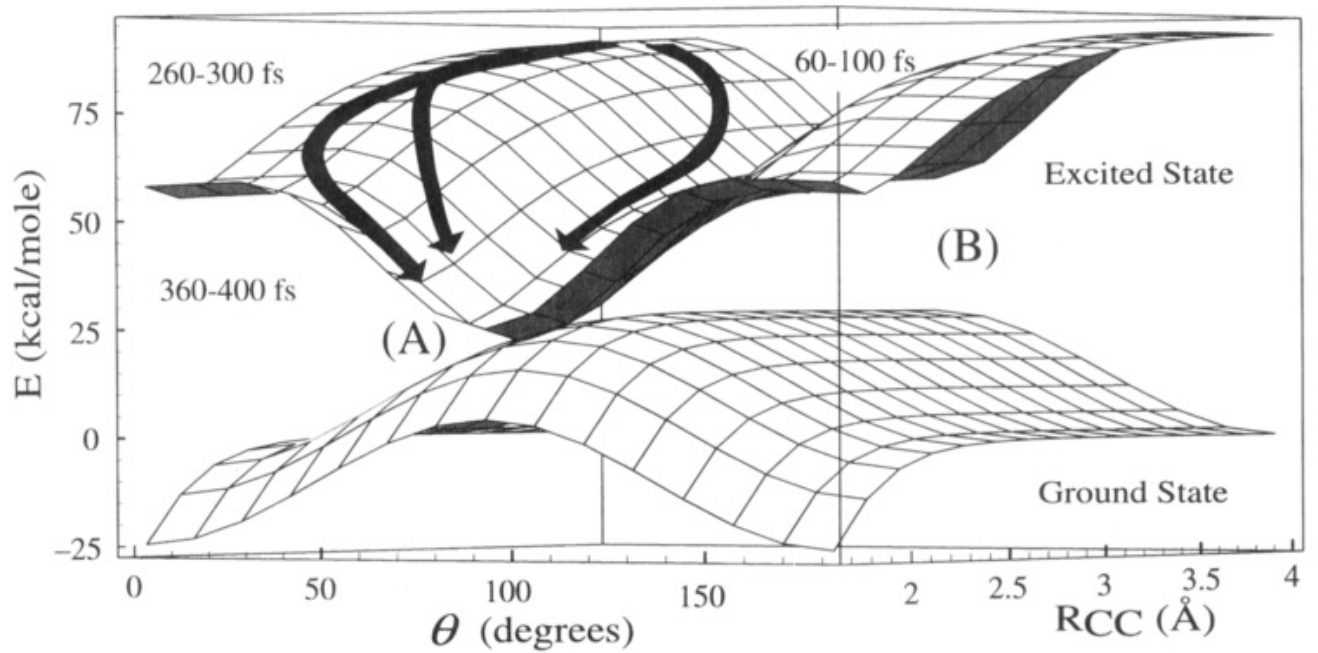

Figure 7. Potential energy surfaces for the $S_{0}$ and $S_{1}$ states of stilbene as a function of the ethylenic torsional angle $(\theta)$ and the interatomic distance between $\mathrm{C}_{2}$ and $\mathrm{C}_{2^{\prime}}\left(R_{\mathrm{CC}}\right)$. Three pathways for the excited state dynamics of photoexcited cis-stilbene are indicated corresponding to the different decay lifetimes observed in the quasiclassical trajectory simulations. The energy gap between the two surfaces has been adjusted to reproduce the rapid internal conversion inferred from ultrafast experiments.

The rapid decay, occurring between 60 and $100 \mathrm{fs}$, appears to be due to early crossing of the ethylenic torsion barrier to the perpendicular $\left(\theta=90^{\circ}\right)$ configuration. This is followed by a fast decrease in the $\mathrm{C}_{2}-\mathrm{C}_{2}$ interatomic distance (the photocyclization coordinate). The middle group of trajectories, whose decay times are close to the ensemble average, first moves toward smaller $\mathrm{C}_{2}-\mathrm{C}_{2}$ ' distances and then "slides" across the potential surface toward the minimum at $\theta=90^{\circ}$. Finally the slow time scale group of the ensemble follows the photocyclization coordinate for a longer time before undergoing ethylenic torsion and requires between 360 and $400 \mathrm{fs}$ to reach the potential minimum at $\theta=90^{\circ}$. These pathways are roughly indicated on a surface plot of the excited state potential in Figure 7.

Motion in the potential-minimum region of the excited state is characterized (see Figure 5 for $t>400 \mathrm{fs}$ ) by torsional oscillations around the $\theta=90^{\circ}$ position with an amplitude of $\approx 10^{\circ}$. In addition, the system oscillates around $R_{\mathrm{C}_{2} \mathrm{C}_{2}} \approx 2 \AA$ with an amplitude $\approx 0.1 \AA$ and the angle $\alpha$ is oscillating about $120^{\circ}$ with an amplitude $\approx 10^{\circ}$ in this region of the potential. One should keep in mind that all 72 vibrations are included in these simulations and that minor variations in the energies and amplitudes of the high-frequency modes occur among different members of the trajectory ensemble. By focusing on the lowfrequency modes which are most important to the isomerization, we nevertheless find that all trajectories in the ensemble pass through the same "intermediate" region of the excited state potential, corresponding to $\theta \approx 90^{\circ}, R_{\mathrm{C}_{2} \mathrm{C}_{2^{\prime}}} \approx 2 \AA, \alpha \approx 120^{\circ}$, and $\phi \approx 20^{\circ}$, before undergoing internal conversion back to the ground electronic state.

An important question is how well does the ensemble of 300 classical trajectories represent the excited state dynamics. Typically, thousands of trajectories are necessary to obtain quantitatively accurate results for large molecular systems using classical simulations. ${ }^{62}$ To determine roughly the convergence properties and qualitative accuracy of our observations, we examined trajectory ensembles of different sizes. Figure 8 shows the distribution of the decay times for (a) 20 trajectories and (b) 100 trajectories. Clearly, the same qualitative features of the distribution are present in all three pictures, Figure 6 and $8 \mathrm{a}, \mathrm{b}$, indicating that the multiple pathway dynamics does not arise from insufficient sampling. We note that similar results are obtained for model potentials with slightly different parameters, but with the same qualitative feature that a small barrier
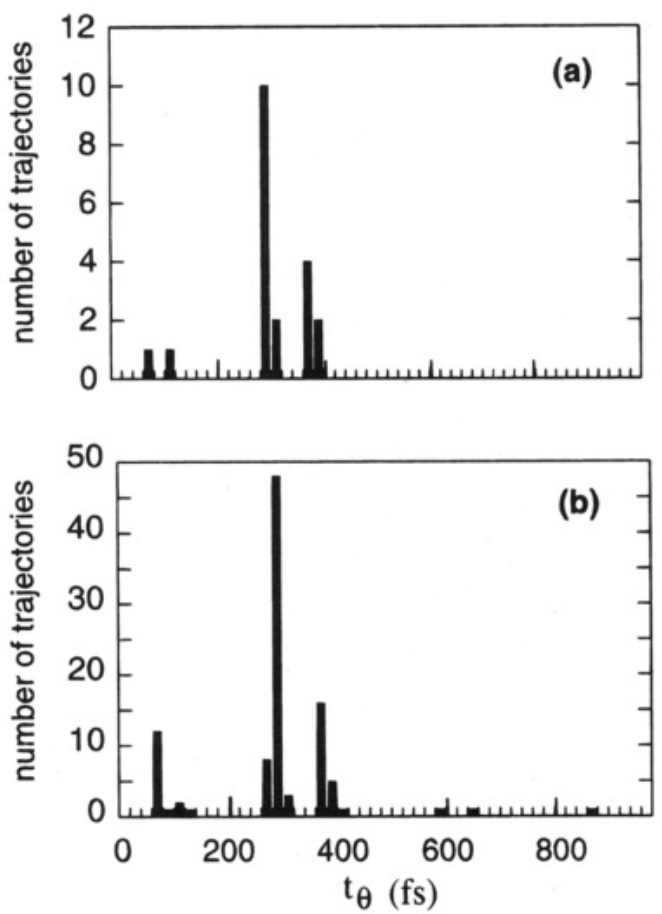

Figure 8. Same as Figure $6 \mathrm{~b}$ for 20 trajectories (a) and 100 trajectories (b).

to cis-trans isomerization is present along the $\theta$ coordinate. Without this barrier, all trajectories follow the same pathway as the rapid decay trajectories, but with a net decay time of only about $30 \mathrm{fs}$.

The use of time-resolved experimental methods to monitor lifetime behavior in molecular systems leads to some interesting consequences. In some sense, such experiments measure the expectation value of the density operator, $\varrho(t)$, evaluated over a probe state, $\langle\phi|\hat{\varrho}(t)| \phi\rangle$. Since the density operator will typically correspond to a mixed state, the experiment "observes" the average behavior of the system. If all the individual molecules in a system follow more or less the same reaction pathway under very similar conditions, then one might observe a narrow distribution of reaction times centered around an average reaction time (most typical for a barrierless process) or an exponential decay for unimolecular processes having a large activation barrier along the reaction path. Interestingly, the 


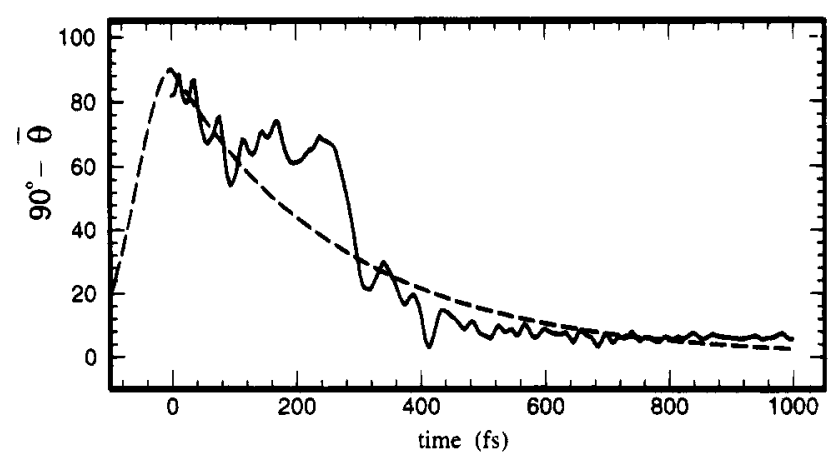

Figure 9. Averaged function $f(\theta)=90^{\circ}-\bar{\theta}(t)$ for the trajectory ensemble (solid line), compared with a single exponential decay (dashed line) with a $280 \mathrm{fs}$ decay parameter. The oscillations are a consequence of the nonstatistical distribution shown in Figure 6.

present case does not appear to fall into either of these categories, instead displaying what we shall term "nonstatistical" lifetime behavior due to the small barrier and multiple-pathway nature of the excited state dynamics. Since the observation of "decay" out of the Franck-Condon region of the $S_{1}$ surface is most sensitive to dynamics along the ethylenic torsion coordinate, we can simulate the expected deviations from exponential decay behavior by examining the averaged time dependence of $\theta$ over the trajectory ensemble (Figure 5a). For clarity, we have plotted in Figure 9 the function $90^{\circ}-\bar{\theta}(t)$ versus time and superimposed an exponential decay curve with a time constant $\tau=280 \mathrm{fs}$. As this figure demonstrates, the net effect of having multiple dynamical pathways and time scales is to impose oscillations on top of an exponential decay curve.

Similar nonexponential decay curves for stilbene in the gas phase have been observed by Pedersen et al ${ }^{10}$ in experiments with 80 fs time resolution. Those workers employed a heuristic model that incorporated an additional cosine term superimposed on the exponential decay curve (see Figure 2 and eq 1 of ref 10) to reproduce their observations. However, in light of the present results, we suggest that the observed behavior is due to decay dynamics that follows multiple pathways on the excited state potential surface. Although 100 trajectories appears to be sufficient to qualitatively represent the excited state dynamics, the larger ensemble used here gives better averaged results and provides an easier comparison with experimental decay curves.

\section{Coupling with the Ground State and Formation of Photoproducts}

We now consider the details of the nonadiabatic dynamics during interconversion back to the ground electronic state. Because all of the dynamics originating in the Franck-Condon region of cis-stilbene proceeds through the same intermediate region on the excited state surface, the branching between the various photoproducts of cis-stilbene is apparently connected with the internal conversion dynamics of the system. To maintain as simple a picture of the nonadiabatic dynamics as possible, we focus attention on the role of the coordinates most closely associated with product formation: the ethylenic torsion

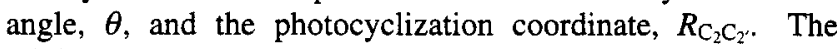
minimum energy separation between the two electronic states along these two coordinates is $\Delta E=4.226 \mathrm{kcal} / \mathrm{mol}(1478$ $\mathrm{cm}^{-1}$ ) and occurs at $\theta=90^{\circ}$ and $R_{\mathrm{C}_{2} \mathrm{C}_{2^{\prime}}}=2.0 \AA$. Throughout the region defined by $79^{\circ}<\theta<101^{\circ}$ and $1.84 \AA<R_{\mathrm{CC}}<$ $2.23 \AA$, the energy separation between the two surfaces comes from the other optically active modes because of the displacements along the corresponding normal coordinates; however, it is in this fairly localized region that the system undergoes nonadiabatic dynamics leading to internal conversion.
Nonadiabatic transitions cannot generally be treated using a purely classical description; however, semiclassical approaches for treating such processes have been developed by Tully and Preston, ${ }^{63}$ and Miller and George. ${ }^{64}$ Initially, the so-called "surface hopping" model was developed primarily to treat problems with very localized regions of strong nonadiabatic coupling. ${ }^{63}$ More recently, it has been modified both to treat problems with extended regions of coupling and to minimize the occurrence of recrossing nonadiabatic trajectories. ${ }^{65}$ The resulting stochastic switching algorithm ${ }^{65}$ minimizes computational effort, simplifies final averaging, and is easy to incorporate in classical molecular dynamics calculations. For a twoelectronic-state system $|0\rangle$ and $|1\rangle$, the method consists of first integrating the classical mechanical equations of motion for the nuclei, then quantum mechanically propagating the electronic wave function along the calculated piece of trajectory, and determining the probabilities $a_{j j}^{2}$ for the system to be in each of the electronic states $\langle j\rangle(j=0,1)$. Switching from one surface to the other occurs if the switching probability is larger than a generated random number between 0 and 1 .

The simplicity of the method makes it widely applicable; however, for large systems the procedure for the electronic part of the model is not feasible. In the spirit of the surface hopping model, use of an appropriate expression for the probability of transition is sufficient to supplement the classical part of the model. Using first-order perturbation theory, the probability of making a transition at moment $\tau$ is given by ${ }^{64}$

$\left.P_{10}(\tau)=\left(\int_{0}^{\tau}\left[\left\langle\psi_{0}\right| \frac{\partial \psi_{1}}{\partial t}\right) a_{1}(t) \exp \left(-\frac{\mathrm{i}}{\hbar} \int_{0}^{t} \Delta E_{10} \mathrm{~d} t^{\prime}\right)\right] \mathrm{d} t\right)^{2}$

where $\psi_{0}$ and $\psi_{1}$ are the electronic wave functins of the ground and excited states and $\Delta E_{10}=E_{1}-E_{0}$ is the time-dependent potential energy difference. Semiclassical evaluation of the time integral gives ${ }^{64}$

$$
P_{10}(\tau)=\exp \left\{-\frac{4 \Delta E_{10}(\tau)}{3 \hbar}\left[\frac{2 \Delta E_{10}(\tau)}{\left(\mathrm{d}^{2} \Delta E_{10} / \mathrm{d} t^{2}\right)_{t=\tau}}\right]^{1 / 2}\right\}
$$

As an example of the semiclassical nonadiabatic procedure, Birge et al. ${ }^{66}$ have used eq 21 in a one-dimensional model of rhodopsin isomerization. They calculate a single trajectory in the excited state, and according to eq 21 (adapted for the onedimensional model), if $P_{10}(\tau)$ is nonzero, the trajectory is split into two pathways, one switching to the ground state and the other continuing in the excited state, meanwhile keeping track of probability weights attached to each pathway. This procedure is followed until the excited state is substantially (97\%) depopulated. Each of the pathways in the ground state is followed until relaxation to trans or cis product is reached, and the quantum yields are calculated by summation of the weights assigned to each branch.

We employ a modification of this algorithm to model the nonadiabatic dynamics of cis-stilbene using an ensemble of classical trajectories on a pair of multidimensional potential surfaces. As outlined above, the quasiclassical ensemble of trajectories are propagated until they enter the region of the potential where internal conversion can occur. Within this region, at each time step, the probability of a nonadiabatic transition is calculated using eq 21 , with the derivatives evaluated numerically. A random number $\xi$ is generated $(0 \leq$ $\xi \leq 1)$, and if $P_{10}(\tau)>\xi$, a switch is invoked to the ground state surface. When this happens for a given trajectory, the momenta are scaled for energy conservation, and the trajectory is continued, now on the ground state potential energy surface, 
until the system has "relaxed" into one of its product minima on the ground state. The relaxation in this case is simply the redistribution of vibrational energy in the molecule (IVR). The final quantum yields for the process are obtained by summing the results over all the trajectories. For each trajectory the total energy is conserved within $0.1 \%$ of the initial value.

This method is particularly well suited for models like the present one which have well-defined nonadiabatic coupling regions. ${ }^{63-65}$ It has also been suggested that, in molecular applications, any simple Landau-Zener-type formula could be used to evaluate the probability of transition or that one could simply invoke nonadiabatic switching when the coupling region is reached. ${ }^{63}$ In the present system, however, the point in phase space at which switching occurs plays an important role in determining the subsequent "relaxation" in the ground state and the final disposition of photoproducts in the reaction. The use of eq 21 is therefore helpful in appropriately sampling the phase space for the switching point in the coupling region.

In the present simulation an ensemble of 300 classical trajectories is used, with initial conditions representing excitation by a 100 fs Gaussian laser pulse with central frequency $\omega_{\mathrm{L}}$ set to $4000 \mathrm{~cm}^{-1}$ excess vibrational energy. The transition probability is calculated via eq 21 at each time step; however, its value becomes significant only after the system surmounts the local barrier at $\theta_{\mathrm{b}} \approx 22^{\circ}$ (at time $t=t_{\theta}$ ) on the excited state surface and the region of strong coupling is reached. With the present surface-switching algorithm, potential energy surfaces, and classical nuclear dynamics, transitions to the ground state take place between 10 and $70 \mathrm{fs}$ after $t=t_{\theta}$. The distribution of the times $t_{\theta}$ was given in section III (see Figure 6). The energy difference between the two electronic states at the time of internal conversion is between 0.15 and $8.0 \mathrm{kcal} / \mathrm{mol}$ for these trajectories; consequently the velocity corrections are small and the inaccuracy associated with using instantaneous transitions (surface hops) instead of some effective potential in the region of transition, should not be substantial. The region in which these transitions occur corresponds to $70^{\circ}<\theta<102^{\circ}$ and 1.92 $<R_{\mathrm{CC}}<2.05 \AA$. In the ground state the "relaxation" occurs to one of three photoproducts: trans-stilbene, cis-stilbene, or DHP with quantum yields of $20.3 \%, 71.7 \%$, and $8.0 \%$, respectively.

All of the trajectories in the ensemble are reactive, that is, they all lead to a transition to the ground state and product formation. The quantum yields in gas phase are not known, and we have adjusted the parameters of the surfaces, specifically those that govern the energy gap between $S_{0}$ and $S_{1}$, to obtain numbers similar to the experimental results $(10 \% \mathrm{DHP})$ for quantum yields of cis-stilbene photoisomerization in methylcyclohexane-isohexane solvent. ${ }^{22}$ The averaged time dependencies of the torsional angle $\theta$, the distance between the $C_{2}$ and $\mathrm{C}_{2^{\prime}}$ atoms, and the bend angle $(\alpha)$ are shown in Figures 10-12 for each of the three photoproduct channels: cis $\rightarrow$ DHP, cis $\rightarrow$ trans, and cis $\rightarrow$ cis. The dynamics indicate that the branching is strongly dependent on both the features of the ground state PES at the point of surface switching and the momenta of the system when switching occurs. Results for individual product channels are summarized below.

DHP Channel Dynamics. The formation of dihydrophenanthrene (DHP) from photoexcited cis-stilbene follows two predominant pathways. Almost two-thirds (65\%) of the trajectories leading to this photoproduct belong to the fast decay pathway, overcoming the ethylenic torsional barrier in 65-90 fs. As a result, the initial motion for these systems is along the cis-trans isomerization coordinate and leads to a torsional angle of $\theta \approx 90^{\circ}$ and $\mathrm{a} \mathrm{C}_{2}-\mathrm{C}_{2^{\prime}}$ distance of $R_{\mathrm{CC}} \approx 2.6 \AA$. This region
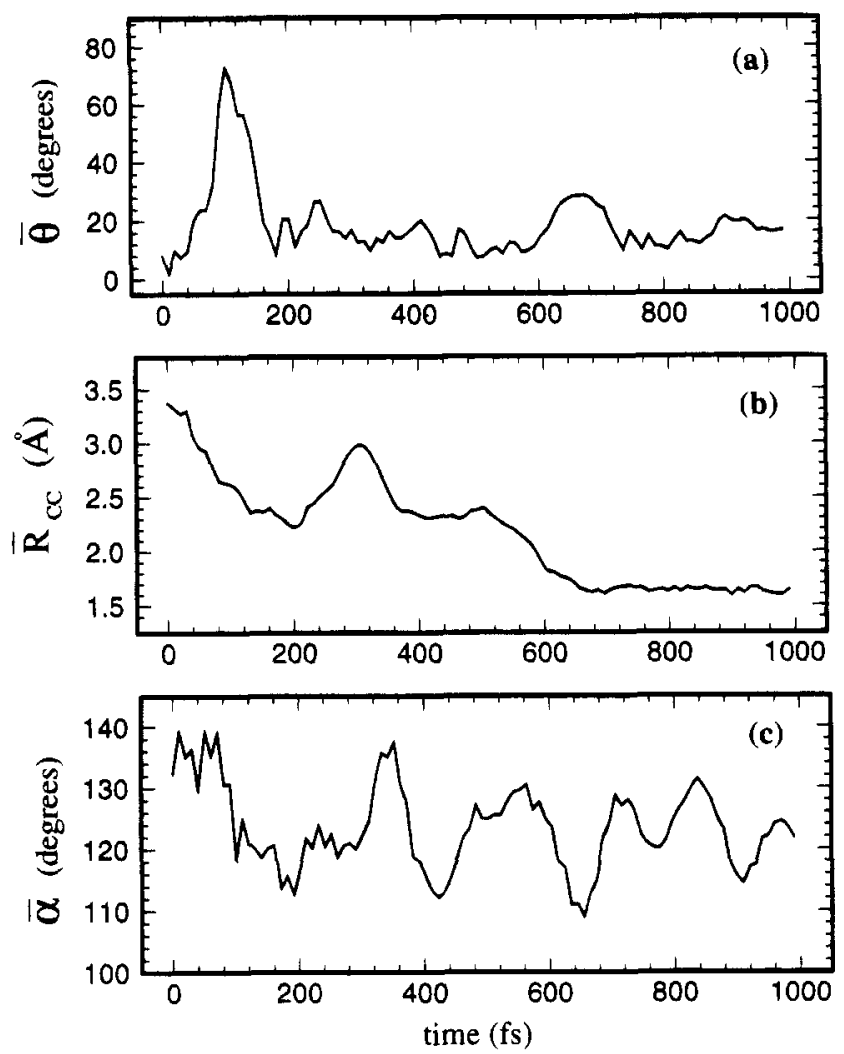

Figure 10. Formation of DHP from excited cis-stilbene. Averaged dependence on time of (a) the ethylenic torsional angle $(\theta)$, (b) the interatomic $\mathrm{C}_{2}-\mathrm{C}_{2^{\prime}}$ distance $\left(R_{\mathrm{CC}}\right)$, and $(\mathrm{c})$ the bending angle $(\alpha)$ for the trajectories leading to DHP formation. The simulations are in the full coordinate space (72 vibrations), but these three coordinates are choosen to represent the reaction pathways.

of the potential has a fairly steep slope along the $R_{\mathrm{C}_{2} \mathrm{C}_{2}}$ coordinate, and the dynamics proceeds to the nonadiabatic transition region by moving to smaller distances $R_{\mathrm{C}_{2} \mathrm{C}_{2}}$. These trajectories typically "hop" to the ground state surface after another 45 fs or so, but do so moving in the direction of smaller $\mathrm{C}_{2}-\mathrm{C}_{2^{\prime}}$ distances, thus favoring photocyclization upon arrival on the ground electronic state. Once the system crosses to the ground state, it further "relaxes" for about $500 \mathrm{fs}$ until $R_{\mathrm{C}_{2} \mathrm{C}_{2}} \approx$ $1.53 \AA$ and the new bond creating the fused ring system is formed.

One explanation for the fast transition to the twisted intermediate in the excited state could be a repulsive van der Waals interaction between the hydrogen atoms on the two phenyl rings, which assists torsion motion toward the perpendicular configuration. In Figure 13, the repulsion energy between $\mathrm{H}_{2}$ and $\mathrm{H}_{2}$ and the torsional angle calculated along a single representative trajectory are shown. Apparently, the increase of the repulsive interaction is followed by a significant increase in the torsional angle. We note, however, that this mechanism should apply only to the early-time dynamics. After about $100 \mathrm{fs}$ the $\mathrm{H}_{2}-\mathrm{H}_{2^{\prime}}$ van der Waals interaction is minimized and the ethylenic torsion $(\theta)$, central bend $(\alpha)$, twist $(\phi)$, and $\mathrm{C}_{2}-\mathrm{C}_{2}$, stretching motions play a more central role in the excited state dynamics. From Figure 13, it is also clear that DHP is formed in the anti configuration for the $\mathrm{H}_{2}$ and $\mathrm{H}_{2}$ atoms which is energetically more favorable. ${ }^{33}$

For the remainder ( $35 \%$ ) of the DHP-forming trajectories, $t_{\theta}$ is from 600 to $800 \mathrm{fs}$, with a nonadiabatic transition occurring after an additional $15 \mathrm{fs}$ or so. At the time of torsional barrier crossing, the $R_{\mathrm{C}_{2} \mathrm{C}_{2}}$ distance has shortened to about $1.95 \pm 0.05$ $\AA$, indicating that the initial motion of the system in this case is along the $R_{\mathrm{C}_{2} \mathrm{C}_{2^{\prime}}}$ coordinate, but the potential has a very gentle 

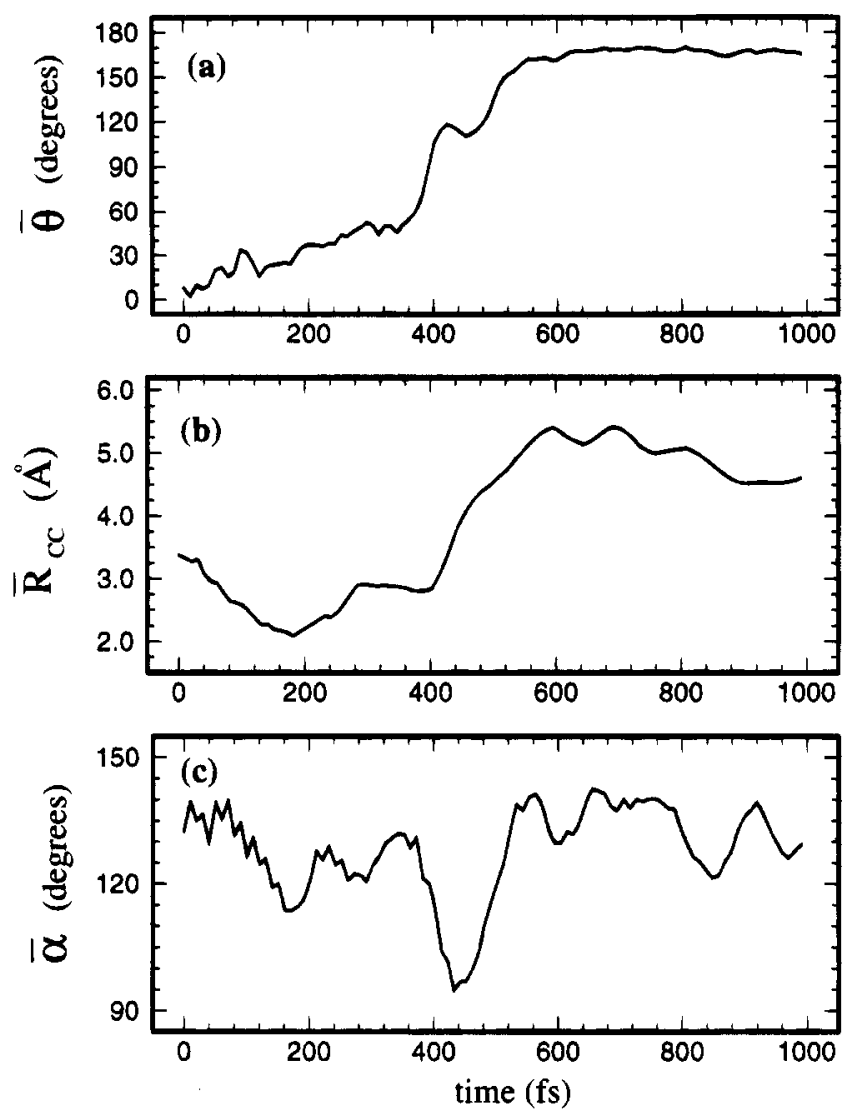

Figure 11. Same as Figure 10 for the formation of trans-stilbene.
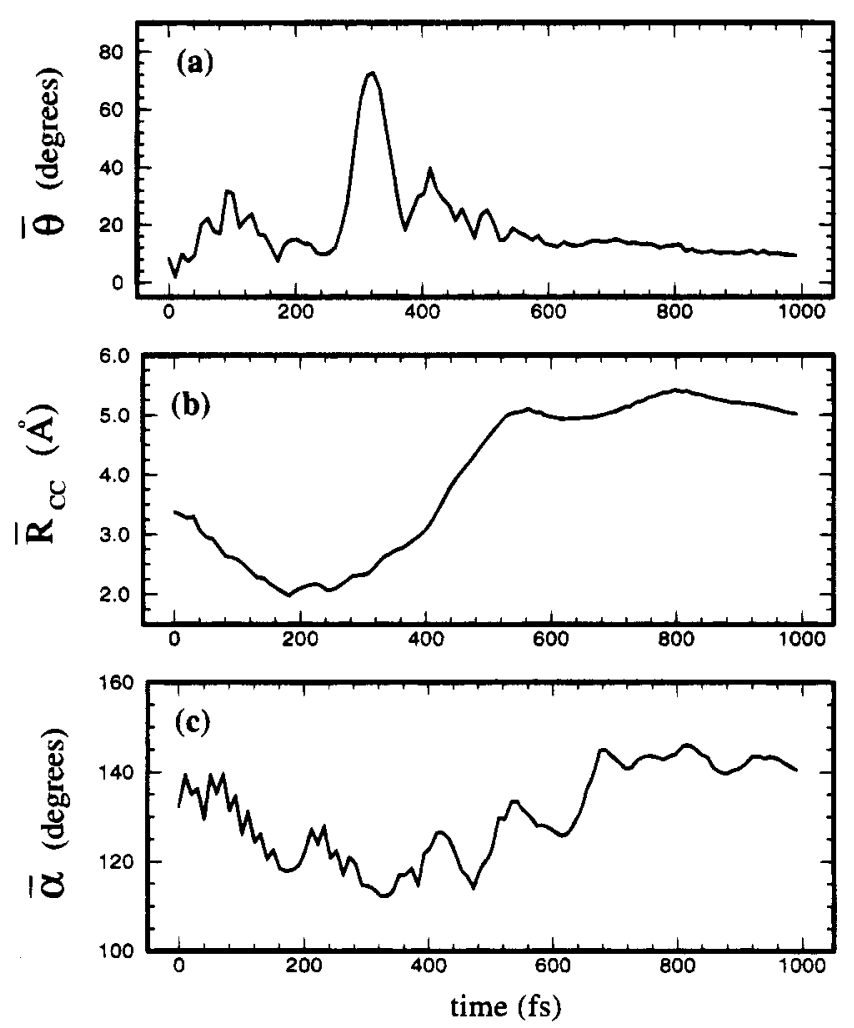

Figure 12. Same as Figure 10 for the formation of ground state cisstilbene.

slope. In contrast to the first group of trajectories, the formation of DHP is dictated by the potential in the ground state rather than the momentum of the system. The "relaxation" of $R_{\mathrm{C}_{2} \mathrm{C}_{2}}$ ' from about 2.0 to $\approx 1.53 \AA$ to form the bond $\mathrm{C}_{2}-\mathrm{C}_{2}$ ' takes about $100 \mathrm{fs}$ for this group of trajectories.
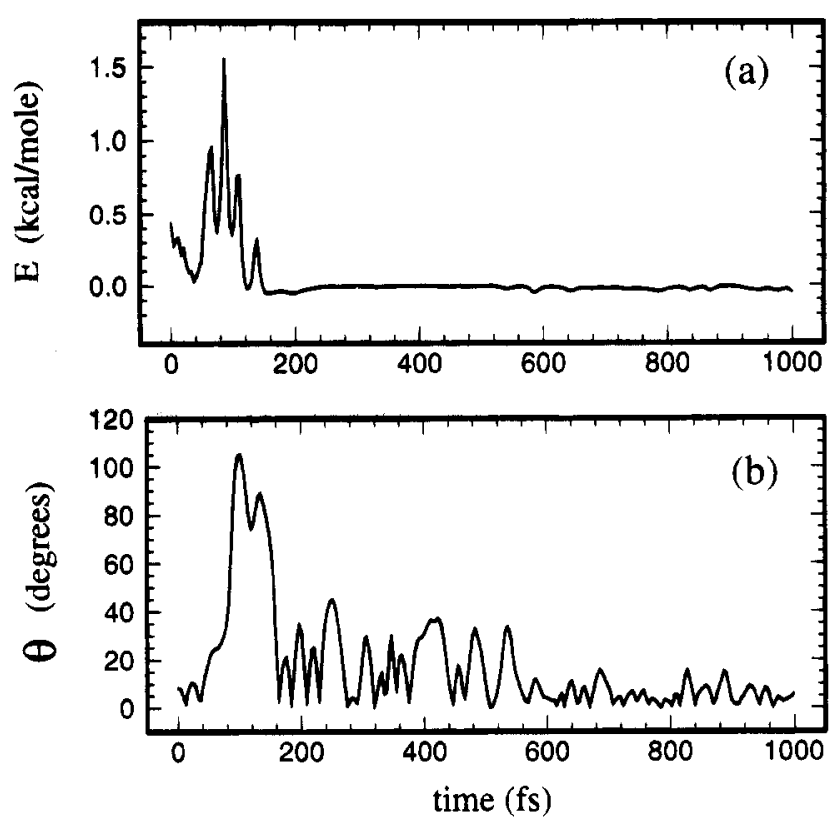

Figure 13. (a) $\mathrm{H}_{2}-\mathrm{H}_{2^{\prime}}$ van der Waals interaction energy calculated along a representative MD trajectory; (b) torsional angle dependence on time along the same trajectory. The increase of the torsional angle follows the increase of the repulsive interaction.

On average, the trajectories leading to DHP formation cross the torsional potential barrier after 273 fs and then undergo radiationless transition to the ground state about $311 \mathrm{fs}$ after photoexcitation (see Figure 10). These averages are very misleading, though, given that the vast majority of these trajectories are either much faster or much slower than this. Both predominant pathways to DHP formation exhibit very short lifetimes in the intermediate region corresponding to the perpendicular configuration, ranging from 15 to $45 \mathrm{fs}$ with an average of about $38 \mathrm{fs}$. At the time of internal conversion, both groups of trajectories have a torsion angle, $\theta \approx 80^{\circ}$; however, the fast group jumps to the ground state surface with $R_{\mathrm{C}_{2} \mathrm{C}_{2}} \approx$ $2.6 \AA$, while the slow group does this at $R_{\mathrm{C}_{2} \mathrm{C}_{2}} \approx 2.0 \AA$. Thus, the dynamics of DHP formation in the photoisomerization of cis-stilbene cannot be generalized easily to any single predominant mechanism.

trans-Stilbene Channel Dynamics. For trajectories leading to trans-stilbene formation, there is again a contribution from two pathways on slightly different time scales; however, the averaged results are more indicative of the dynamics followed by the system on the way to the trans-stilbene region on the ground state (see Figure 11). The average behavior is characterized by crossing the torsional barrier at $t_{\theta}=336 \mathrm{fs}$ and undergoing internal conversion at $t_{0-1}=355$ fs after photoexcitation. Two-thirds $(66 \%)$ of these trajectories are slower than the average taking between 370 and 390 fs to leave the FranckCondon region. One feature common to all trajectories is that, after overcoming the local torsion barrier in the excited state, the movement along $\theta$ is direct to the trans-isomer. Radiationless transitions to the ground state occur at torsional angles of between $70^{\circ}$ and $95^{\circ}$ (average $\approx 81^{\circ}$ ), but for most of the trajectories $\theta_{0-1}<90^{\circ}$ and therefore the isomerization is initially dictated by the system's momentum. After the transition, $\theta$ increases with small oscillations to the trans region of the ground state in about $100 \mathrm{fs}$. The distance between $\mathrm{C}_{2}$ and $\mathrm{C}_{2^{\prime}}$ at the point of the nonadiabatic transition is $2.1 \pm 0.2 \AA$ and increases to the typical trans-stilbene conformation distance of $R_{\mathrm{C}_{2}} \mathrm{C}_{2^{\prime}} \geq$ $5 \AA$ in $150-200$ fs. Since the initial increase of the torsional angle is due to changes in the positions of the atoms at the 


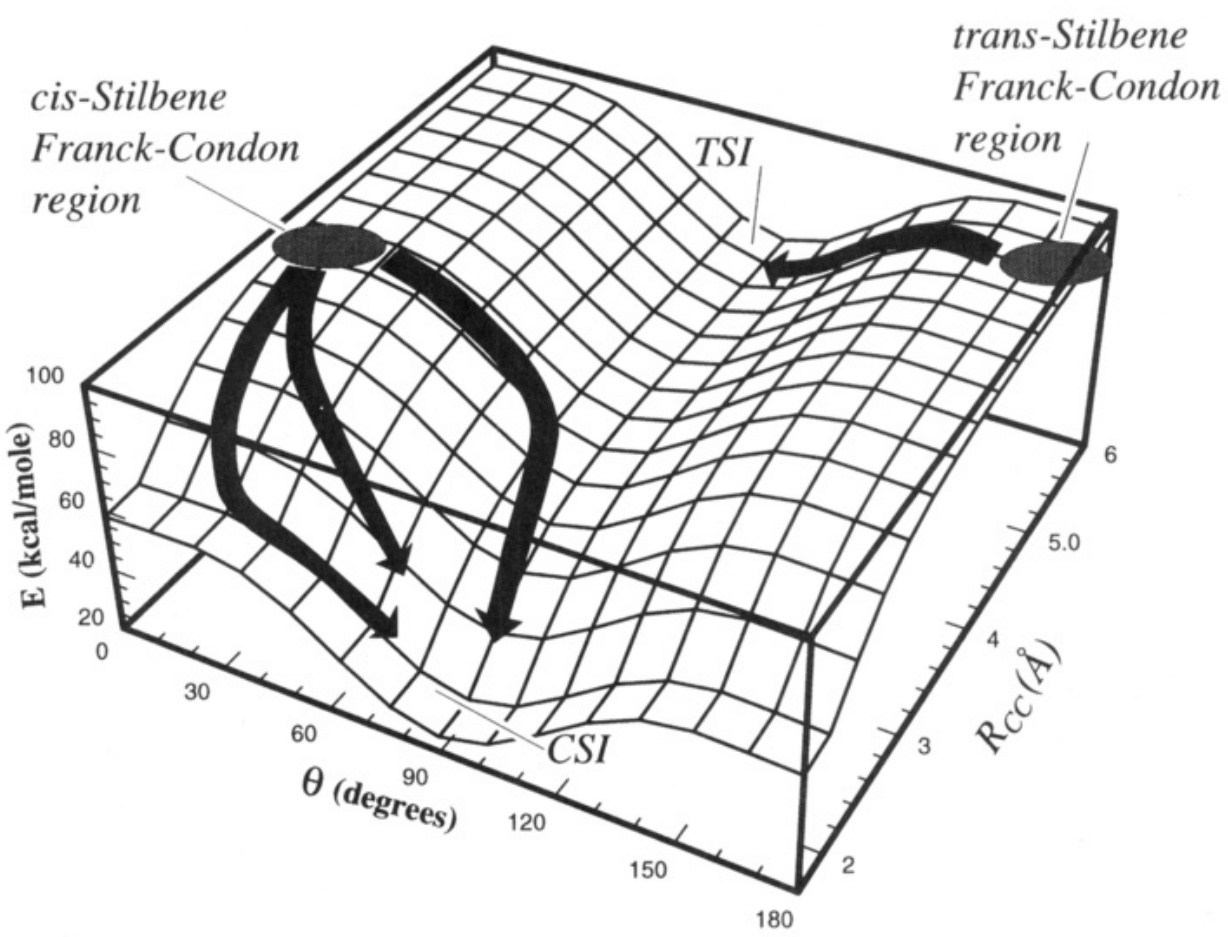

Figure 14. Comparison of the dynamics of excited trans- and cis-stilbene showing transitions to different minima on the $\mathrm{S}_{1}$ surface, respectively, trans-stilbene intermediate (TSI) and cis-stilbene intermediate (CSI), although they both correspond to a perpendicular configuration of the torsional coordinate $\left(\theta=90^{\circ}\right)$.

ethylenic bridge, this is also approximately the time required for the phenyl rings to follow the torsional motion.

cis-Stilbene Channel Dynamics. In the present simulations, a majority of the trajectories leads to re-formation of cis-stilbene on the ground state surface. Unlike the formation of DHP, the average behavior of trajectories in this group, with $t_{\theta}=265 \mathrm{fs}$ and internal conversion occurring about $286 \mathrm{fs}$ after photoexcitation, is representative of the group as a whole. For almost three-quarters $(72 \%)$ of the trajectories, $t_{\theta}$ is between 260 and $320 \mathrm{fs}$, and all of the trajectories in the ensemble with $t_{\theta}$ in this range (see Figure 6) belong to the cis-stilbene channel. Radiationless transitions to the ground state occur at torsional angles of between $70^{\circ}$ and $102^{\circ}$ and $\mathrm{C}_{2}-\mathrm{C}_{2}$ ' distances of about $2.1 \AA$, although most of the trajectories undergo the surface hop with $\theta_{0-1}<90^{\circ}$. After the switching, the torsional angle increases up to $100-110^{\circ}$ as if to form trans-stilbene, then "relaxes" to the $0^{\circ}$ cis region in about $100 \mathrm{fs}$.

It is interesting to note that the averaged results for both the time scale of decay and the point in phase space at which internal conversion occurs are very similar for all three channels. Nevertheless, the branching among the channels is not statistical and there are specific dynamical features that are manifested in the averaged time dependence of the primary isomerization coordinates. In particular, the correlation between the time scale for decay out of the Franck-Condon region and the eventual photoproduct formed is interesting. As we have noted, the ensemble can be roughly divided into three groups corresponding to fast, average, and slow decay times. Almost two-thirds of the DHP-forming tranjectories come from the fast group, twothirds of the trans-stilbene-forming trajectories belong to the slow group, and all of the "average" group of trajectories lead to cis-stilbene formation. After these trajectories make nonadiabatic transitions to the ground state, the system requires an additional $500 \mathrm{fs}$ to "relax" to the DHP minimum, 150-200 fs to form ground state trans-stilbene, and only 100 fs to re-form cis-stilbene.

Photoisomerization of trans-Stilbene. The differences between cis- and trans-stilbene and their associated photo- isomerization reactions are striking. Photoexcitation of cisstilbene leads to a variety of products formed on a relatively short time scale $(\approx 300 \mathrm{fs})$, while the corresponding process in trans-stilbene produces a near 50:50 cis:trans branching ratio and requires a much longer time $(\approx 80 \mathrm{ps})$. Much of the difference can be attributed to the presence of both the bonding and the repulsive interactions between the phenyl rings in the excited state which can accelerate the isomerization rate and facilitate the formation of the photocyclization product. It is useful to examine these differences between the two isomers in the context of the dynamics that occurs on the excited state after photoexcitation.

One advantage of using a molecular mechanics-type force field is that it permits us to compare the dynamics of cis and trans using the same theoretical model. It is, therefore, interesting to examine the consistency of the model with the trans-stilbene dynamics. However, due to the much longer time scale, modeling the photoisomerization reactions of transstilbene demands a much greater computational effort. As a result, we have restricted our study to a small ensemble of 50 trajectories and simply compare the predominant dynamical pathways to those of cis-stilbene. The trans conformer has a planar minimum in both electronic states, with a distance of about $5.56 \AA$ between the $\mathrm{C}_{2}$ and $\mathrm{C}_{2}$, atoms. At this distance, the bonding interaction between the phenyl rings is effectively zero and plays little or no role in the initial excited state dynamics. The main reaction pathway is along the ethylenic torsion coordinate and involves crossing the local torsional barrier and moving to a perpendicular $\left(\theta=90^{\circ}\right)$ configuration (see Figure 14). Thus, unlike cis-stilbene, the photoisomerization of trans-stilbene appears to be well described by a single degree of freedom.

Some other important differences between the two isomers can also be observed. Perhaps the most surprising is that the perpendicular configuration attained by photoexcited transstilbene (trans-stilbene intermediate, TSI) is different from that reached from the cis side (cis-stilbene intermediate, CSI), although it does correspond to a local minimum in the $S_{1}$ state 

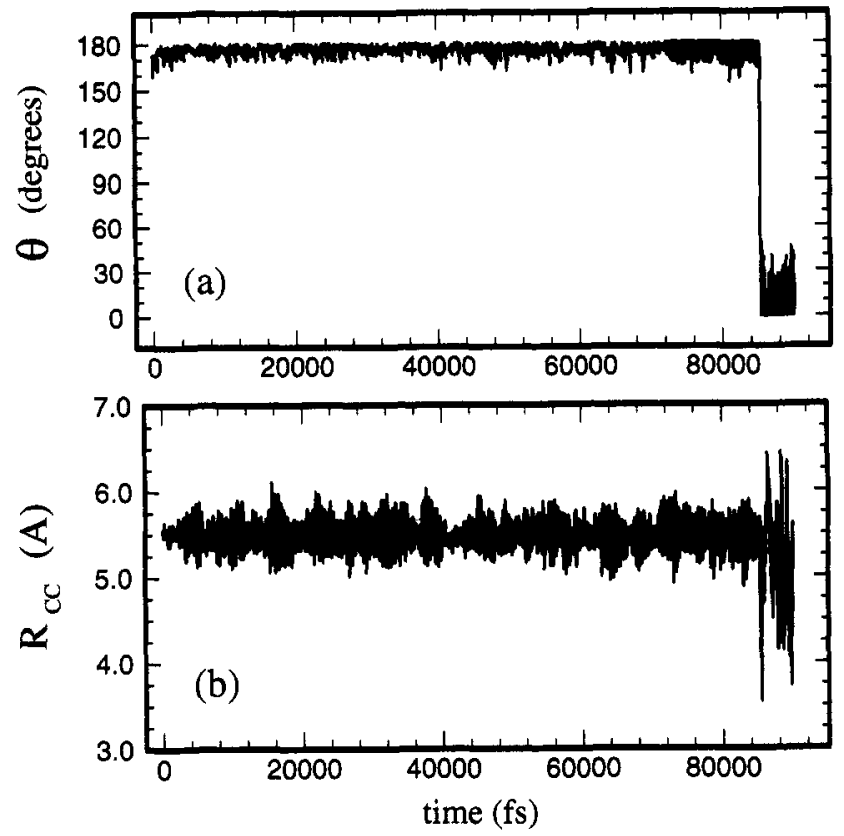

Figure 15. Isomerization of trans-stilbene: time dependence of (a) the ethylenic torsional angle $(\theta)$ and (b) the interatomic $\mathrm{C}_{2}-\mathrm{C}_{2^{\prime}}$ distance, calculated along a representative trajectory to illustrate the difference from the isomerization of cis-stilbene while using the same surfaces (eqs 14-19).

potential (see Figure 14). Subsequent internal conversion from this local minimum leads to vibrational relaxatin to either cisor trans-stilbene in the ground state but does not result in any formation of DHP, consistent with experimental observations. Typical time dependencies of the ethylenic torsional angle and the interatomic $\mathrm{C}_{2}-\mathrm{C}_{2^{\prime}}$ distance are shown in Figure 15 for trans $\rightarrow$ cis isomerization.

Along the torsional coordinate we see rapid oscillations until a sufficient amount of energy is transfered from other degrees of freedom for the barrier to be overcome (Figure 15a). Once this happens, the transition to the twisted intermediate is fast, taking less than 30 fs. However, in contrast to cis-stilbene, the time spent in the TSI region appears to be significantly longer. In the ensemble studied here it is between 30 and 400 fs and, although the size of the ensemble is probably not sufficient to estimate the lifetime of the TSI, it is apparently longer than that of the CSI $(\approx 25 \mathrm{fs})$.

The time dependence of the photocyclization bonding coordinate, $R_{\mathrm{C}_{2} \mathrm{C}_{2}}$, is the main difference in the dynamics compared to those of cis-stilbene (Figure 15b). It is characterized by oscillations with moderate amplitude, while in cis-stilbene it exhibits direct movement on a $300 \mathrm{fs}$ time scale to the CSI. This coordinate is directly related to the bonding interaction between the phenyl rings, and its behavior is not surprising given that the bending mode is the strongest in the absorption spectrum and is also strongly coupled with the other vibrations along the ethylenic bridge. After overcoming the barrier along the torsional coordinate, a substantial portion of the potential energy becomes kinetic energy and redistributes to other degrees of freedom. This is the reason for the increased amplitude of the oscillations along the $R_{\mathrm{C}_{2} \mathrm{C}_{2}}$, coordinate. Further energy redistribution in the ground electronic state leads to relaxation to the $R_{\mathrm{C}_{2} \mathrm{C}_{2}}$, values more typical for cis-stilbene.

\section{Discussion and Summary}

In earlier work, ${ }^{47}$ we studied the photoinduced dynamics of cis-stilbene leading to the twisted (perpendicular) intermediate in the excited state and found compelling evidence for the existence of a barrier along the torsional coordinate. Without a barrier, the average time for leaving the Franck-Condon (FC) region is $30 \mathrm{fs}$, much smaller than the experimentally observed time of approximately $300 \mathrm{fs}$. The presence of a barrier along this coordinate increases the isomerization time, and by matching the decay time of our simulations to experiment, an estimate of between 510 and $640 \mathrm{~cm}^{-1}$ for the torsional barrier could be made. The torsional potential both there and in the present work is modeled as a truncated Fourier series with the barrier determined by the relative values of the coefficients, $V_{2}$ and $V_{4}$, of the first two terms. For $V_{2}$ in the earlier study, we used the value $-7850 \mathrm{~cm}^{-1}$ deduced ${ }^{10}$ from the resonance Raman measurements of Myers and Mathies. ${ }^{39}$ This estimate, however, is made by inferring the slope of the potential in the FranckCondon region and is based on the assumption that the torsional potential is barrierless. When a barrier is present, this value must be modified and here we use a value of $-11597 \mathrm{~cm}^{-1}$. This value also gives a reasonable energy gap between the ground and excited states in the region of the twisted intermediate. Consequently, in the present model, we make an improved estimate for the torsional barrier of $V_{\theta}=340 \mathrm{~cm}^{-1}$ at $\theta_{\mathrm{b}}=$ $22.5^{\circ}$.

On a multidimensional potential energy surface (PES) the barrier along a particular coordinate depends also on the profile of the surface along the other coordinates. Among several activated coordinates on the excited state, the twist motion of the phenyl rings ( $\phi$, see Figure 1$)$ appears to affect the torsional isomerization of stilbene the most. The potential parameters $V_{2}$ and $V_{4}$ for the potential along this coordinate (Table 2) are chosen to give a value for the corresponding barrier between the two estimates available from the literature: $750 \mathrm{~cm}^{-1} 67$ and $1500 \mathrm{~cm}^{-1} .^{12}$ Given this uncertainty in the phenyl twist potential, the estimate for the torsional barrier ranges between 260 and $400 \mathrm{~cm}^{-1}$ but usually occurs at a torsional angle of approximately $22^{\circ}$ when the time scale of the reaction dynamics is adjusted to match experiment.

Our simulations suggest that an ensemble of about 100 trajectories is sufficient to obtain good averaged results for the excited state lifetime, although 300 trajectories are used in the present study. The distribution of decay times in the ensemble does not fit a statistical model (Figures 6 and 8 ) as $84 \%$ of the trajectories fall into one of three narrow groups representing different lifetimes and pathways from the Franck-Condon region to the perpendicular intermediate on the excited state. As a result of these multiple pathways, the simulated isomerization rate of the system is an exponential decay with superimposed oscillations (see Figure 9) and reflects what we consider to be essentially nonstatistical rate behavior. A similar result has been reported experimentally in ultrafast time-resolved measurements of gas phase cis-stilbene. ${ }^{10}$ In large molecular systems, it is likely that such complicated dynamics frequently play a role in the chemical processes of a system and the experimental observation of complex decay curves may generally indicate the presence of multiple reaction pathways. This is a matter requiring further investigation.

After leaving the Franck-Condon region, the molecule is quickly trapped (within $50 \mathrm{fs}$ ) in the region of the twisted (perpendicular) intermediate. Once in this region, the amplitude of the torsional angle oscillations is less than $15^{\circ}$ (Figure 5a) and therefore a return to the Franck-Condon region or direct isomerization to trans-stilbene in $S_{1}$ is not possible. Similar results, namely damped motion along the torsional coordinate, have been reported in a molecular dynamics simulation of rhodopsin ${ }^{68}$ and a quantum simulation of a model fourdimensional cis-trans isomerization system, ${ }^{69}$ indicating that our results probably reflect the multidimensional nature of the 


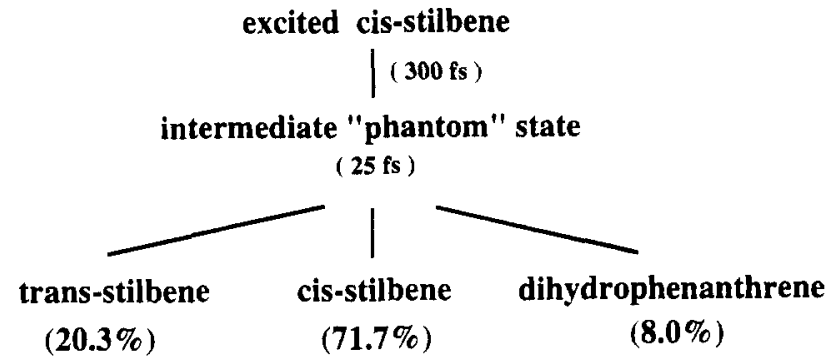

Figure 16. Schematic summary of the present model for cis-stilbene photoisomerization.

problem rather than the particular potential surface used. This contradicts the precise measurement of a small amount $(0.0020$ quantum yield) of trans-stilbene fluorescence which Saltiel and co-workers claim arises from a direct cis* $\rightarrow$ trans* adiabatic conversion ${ }^{70}$ Their observation could be attributed to nonadiabatic excited state dynamics involving a transition to a shallow higher lying excited state and, if so, the small quantum yield reassures us that excited state nonadiabaticity is not important for the present study. Nonadiabaticity in the excited state may, however, be important for higher energy excitation and may explain increased lifetimes reported by Pedersen $e t$ al. for photoexcitation of stilbene using shorter wavelength light. ${ }^{10}$

The present model calculations include three major parts: (i) excited cis-stilbene vibrational dynamics leading to a minimum at the perpendicular configuration on $\mathrm{S}_{1}$ (cis-stilbene intermediate, CSI) (ii) nonadiabatic internal conversion dynamics to the ground electronic state, and (iii) "relaxation" in $\mathrm{S}_{0}$ to the three photoproducts, DHP, trans-stilbene, and cis-stilbene (Figure 16). The excited state vibrational dynamics to the CSI region is characterized by large amplitude movement along both the torsional and $\mathrm{C}_{2}-\mathrm{C}_{2^{\prime}}$ bonding coordinates, which establishes some preliminary conditions for formation of the photoproducts in the ground state. The average lifetime of the system in the CSI region is about $25 \mathrm{fs}$ in accordance with the experimental estimate of $<150 \mathrm{fs}^{3}$ Prior to internal conversion, the excited state part of the reaction pathways is similar to cis $\rightarrow$ DHP and cis $\rightarrow$ trans and this could, in part, account for the similar results from anisotropy measurements of reactant-product orientations for the two products. ${ }^{3}$ The branching between the various photoproducts occurs during the radiationless transition to the ground state and is influenced by the momentum of the system as it enters the surface-hopping region and by the ground state potential surface at the point where the surface hop is made. No differentiation between the product channels appears to be made during the time the system resides on the excited state surface.

Nevertheless, interesting correlations can be made between the excited state dynamics and the eventual formation of the products on the ground state. Specifically, most of the trajectories leading to DHP undergo early internal conversion $\left(t_{\theta} \approx 80 \mathrm{fs}\right)$; all of the trajectories with $t_{\theta} \approx 280 \mathrm{fs}$ lead to formation of cis-stilbene and all with $t_{\theta} \approx 380 \mathrm{fs}$ to transstilbene. Such time separation among reaction channels is the ideal case for applying various schemes to control the molecular dynamics and product formation. Along these lines, one can conceive the possibility of selectively enhancing certain product channels by preparing the ground state molecule in an appropriate vibrationally excited state before electronic excitation: a two-color IR/UV excitation. ${ }^{2}$ For example, if the ethylenic torsion were vibrationally excited before transition to the $S_{1}$ state, it might cause a greater fraction of the molecules to follow the "fast decay pathway" which enhances the eventual formation of DHP. Similarly, excitation of the central bending and phenyl twisting motions before the electronic transition might increase the fraction of molecules following the slower decay pathways on the excited state, thus enhancing the cis- and trans-stilbene product channels. An interesting aspect of the present results is that the net effect of vibrational excitation in the ground state is counterintuitive: by initially exciting the ethylenic torsion (the cis-trans isomerization coordinate), one might enhance the production of photocyclization product and vice-versa. We are presently investigating these possibilities through an extension of the present classical dynamics simulations.

For excited trans-stilbene, the only active coordinate is the ethylenic torsion and the molecule relaxes to a different local minimum on the $S_{1}$ state (trans-stilbene intermediate, TSI), although it also corresponds to a perpendicular configuration of the torsional coordinate. Thus, our model differs from the traditionally accepted picture of a single perpendicular intermediate common to the photoisomerization dynamics of both cis-and trans-stilbene. ${ }^{8}$ As a result, the present model accounts for both the formation of DHP from the photoexcitation of cisstilbene and its absence among the photoproducts of transstilbene. A more detailed study of trans-stilbene excited state dynamics currently underway should provide more insight into the origin of stochasticity (that is, complete IVR) in contrast to the nonstatistical features found for cis-stilbene.

A number of approximations have been used in the present study. Despite the functional simplicity of the potential energy surfaces, they probably provide a reasonably good representation. of the general features of the system, with the possible exception of the $\mathrm{C}_{2}-\mathrm{C}_{2}$ bonding interaction. Our understanding of the photocyclization reaction is much less well developed than for the cis-trans isomerization, and therefore, ultrafast experimental measurements, as well as electronic structure calculations of DHP and the diphenylcycloalkenes, will be very helpful for future studies. Electronic structure calculations ${ }^{27,32 b, 33}$ of DHP ground state structure indicate that electronic rearrangement takes place, and thus, our simulation of the ground state "relaxation" may be reliable only for the initial dynamics leading to formation of the products.

Classical simulations of molecular dynamics are most effective for systems involving a single Born-Oppenheimer surface, and then only if an effective means for simulating quasiclassically an initial quantum nonstationary state is available. We have described such an approach for the case of a finite (nonzero) temperature state prepared on an electronic excited state by an ultrashort laser pulse. Classical studies are less effective for treating systems which exhibit nonadiabatic transitions between electronic states and, although well-established "surface-hopping" algorithms are available to treat these transitions, ${ }^{63-65}$ this represents a weakness in the present study, especially given the apparent importance of the nonadiabatic dynamics in determining the final photoproduct distribution. Continuing studies on this system are aimed at combining quantum and classical approaches to studying the excited state dynamics and using reduced-dimensionality models to represent the competing isomerization processes in a quantum wave packet dynamics study. It is hoped that this way, we might determine any inconsistencies or inaccuracies introduced by using the semiclassical "surface-hopping" algorithm and learn more about the importance of the "bath" vibrations in the molecule.

Acknowledgment. This work has been supported primarily by the U.S. National Science Foundation under Grant CHE9116187 (to J.H.F.). In addition, V.N.Z. acknowledges support from the International Science Foundation under the Grant MS9000. 


\section{References and Notes}

(1) Zewail, A. H. J. Phys. Chem. 1993, 97, 12427.

(2) Crim, F. F. Annu. Rev. Phys. Chem. 1993, 44, 397.

(3) Sension, R. J.; Repinec, S. T.; Szarka, A. Z.; Hochstrasser, R. M. J. Chem. Phys, 1993, 98, 6291.

(4) Heeger, A. J.; Kivelson, S.; Schrieffer, J. R.; Su, W. P. Rev. Mod. Phys. 1988, 60, 781.

(5) Birge, R. R. Annu. Rev. Phys. Chem. 1990, 41, 683.

(6) (a) Akhmanov, S. A.; Vysloukh, V. A.; Chirkin, A. S. Optics of Femtosecond Laser Pulses; Am. Inst. Phys.: New York, 1992. (b) Koroteev, N. I.; Shumay, I. L. Physics of High-Power Laser Radiation; Nauka: Moscow, 1991 (in Russian).

(7) (a) Mathies, R. A.: Cruz, C. H. B.; Pollard, W. T.; Shank, C. V. Science 1988, 240, 777. (b) Shoenlein, R. W.; Peteanu, L. A.; Mathies, R A.; Shank, C. V. Science 1991, 254, 412.

(8) For reviews see (a) Waldeck, D. H. Chem. Rev. 1991, 91, 415 (b) Saltiel, J.; Sun, Y.-P. In Photochromism Molecules and Systems; Dürr H., Bouas-Laurent, H., Eds.; Elsevier: Amsterdam, 1990, p 64. (c) Hochstrasser, R. M. Pure Appl. Chem. 1980, 52, 2683.

(9) Green, B. I,; Farrow, R. C. J. Chem. Phys. 1983, 78, 3336 8801 .

(10) Pedersen, S.; Bañares, L.; Zewail, A. H. J. Chem. Phys. 1992, 97,

(11) (a) Petek, H.; Fujiwara, Y.; Kim, D.; Yoshihara, K. J. Am. Chem. Soc. 1988, 110, 6269. (b) Petek, H.; Yoshihara, K.; Fujiwara, Y.; Frey, J. G. J. Opt. Soc. Am. 1990, B7, 1540 .

(12) (a) Petek, H.; Yoshihara, K.; Fujiwara, Y.; Lin, Z.; Penn, J. H.; Frederick, J. H. J. Phys. Chem. 1990, 94, 7539. (b) Frederick, J. H.; Fujiwara, Y.; Penn, J. H.; Yoshihara, K.; Petek, H. J. Phys. Chem. 1991, $95,2845$.

(13) Todd, D. C.; Jean, J. M.; Rosenthal, S. J.; Ruggiero, A. J.: Yang, D.; Fleming, G. R. J. Chem. Phys. 1990, 93, 8658.

(14) Rice, J. K.; Baronavski, A. P. J. Phys. Chem. 1992, 96, 3559.

(15) Nikowa, L.; Schwarzer, D.; Troe, J.; Schroeder, J. J. Chem. Phys. 1992, $97,4827$.

(16) Todd, D. C.; Fleming, G. R. J. Chem. Phys, 1993, 98, 269.

(17) Doany, F. E.; Hochstrasser, R. M.; Greene, B. I.; Millard, R. R. Chem. Phys. Lett. 1985, $118,1$.

(18) (a) Lee, M.; Haseltine, J. N.; Smith, A. B., III; Hochstrasser, R M. J. Am. Chem. Soc. 1989, 111, 5044. (b) Abrash, S.; Repinec, S. T. Hochstrasser, R. M. J. Chem. Phys. 1990, 93, 1041. (c) Repinec, S. T.; Sension, R. J.; Hochstrasser, R. M. Ber. Bunsen-Ges. Phys. Chem. 1991 95, 248. (d) Sension, R. J.; Repinec, S. T.; Hochstrasser, R. M. J. Chem Phys. 1990, 93, 9185; J. Phys. Chem. 1991, 95, 2946. (e) Sension, R. J.; Szarka, A. Z.; Hochstrasser, R. M. J. Chem. Phys. 1992, 97, 5239.

(19) Repinec, S. T.; Sension, R. J.; Szarka, A. Z.; Hochstrasser, R. M. J. Phys. Chem. 1991, 95, 10380.

(20) Philips, D. L.; Rodier, J.-M.; Myers, A. B. Chem. Phys. 1993, I75,

(21) Mallory, F. B.; Wood, C. S.; Gordon, J. T. J. Am. Chem. Soc. 1964, $86,3094$.

(22) (a) Muszkat, K. A.; Fisher, E. J. Chem. Soc. B 1967, 662. (b) Wismonski-Knittel, T.; Fischer, G.; Fischer, E. J. Chem. Soc., Perkin Trans. I 1974, 1930.

(23) (a) Malkin, S.; Fischer, E. J. Phys. Chem. 1964, 68, 1153. (b) Gegiou, D.; Muszkat, K. A.; Fisher, E. J. Am. Chem. Soc. 1968, 90, 3907. (24) Sharafy, S.; Muszkat, K. A. J. Am. Chem. Soc. 1971, 93, 4119.

(25) (a) Saltiel, J. J. Am. Chem. Soc. 1967, 89, 1036. (b) Saltiel, J. Marinari, A.; Chang, D. W. L.; Mitchener, J. C.; Megarity, E. D. J. Am. Chem. Soc. 1979, 101, 2982

(26) Saltiel, J.; Waller, A. S.; Sears, D. F., Jr.; Garett, C. Z. J. Phys. Chem. 1993, 97, 2516.

(27) (a) Muszkat, K. A.; Schmidt, W. Helv. Chim. Acta 1971, 54, 1195. (b) Muszkat, K. A. In Topics in Current Chemistry; Springer-Verlag: Berlin, 1980, Vol. 88, p 89.

(28) Warshel, A. J. Chem. Phys. 1975, 62, 214.

(29) Olbrich, G. Ber. Bunsen-Ges. Phys. Chem. 1982, 86, 209.

(30) Troe, J.; Weitzel, K.-M. J. Chem. Phys. 1988, 88, 7030.

(31) Rettig, W.; Strehmel, B.; Majenz, W. Chem. Phys. 1993, 173, 525.

(32) (a) Negri, F.; Orlandi, G.; Zerbetto, F. J. Phys. Chem. 1989, 93,

5124. (b) Negri, F.; Orlandi, G. Chem. Phys. Lett. 1992, 195, 523.

(33) Zhou, X.; Liu, R. Chem. Phys. Lett. 1992, 191, 477.
(34) Syage, J. A.; Felker, P. M.; Zewail, A. H. J. Chem. Phys. 1984, 81,4685 .

(35) Suzuki, T.; Mikami, N.; Ito, M. J. Phys. Chem. 1986, 90, 6431. 2782 .

(37) Urano, T.; Hamaguchi, H.; Tasumi, M.; Yamanouchi, K.; Tsuchiya K.; Gustafson, T. L. J. Chem. Phys. 1989, 91, 3884.

(38) Champagne, B. B.; Pfanstiel, J. F.; Plusquellic, D. F.; Pratt, D. W.; Herpen, W. M.; Meerts, W. L. J. Phys. Chem. 1990, 94, 6.

(39) Myers, A. B.; Mathies, R. A. J. Chem. Phys. 1984, 81, 1552.

(40) Rodier, J.-M.; Ci, X.; Myers, A. B. Chem. Phys. Lett. 1991, I83,

(41) (a) Kamalov, V. F.; Koroteev, N. I.; Shkurinov, A. P.; Toleutaev, B. N. Chem. Phys. Lett. 1988, 147, 335. (b) Kamalov, V. F.; Koroteev, N I.; Toleutaev, B. N.; Shkurinov, A. P.; Stamm, U. J. Phys. Chem. 1989 $93,5645$.

(42) (a) Momicchioli, F.; Baraldi, I.; Bruni, M. C. J. Chem. Soc., Faraday Trans. 2 1972, 68, 1556; (b) Chem. Phys. 1983, 82, 229. 8915.

(44) Gruner, D.; Brumer, P.; Shapiro, M. J. Phys. Chem. 1992, 96, 281. 755 .

(46) Syage, J. A.; Felker, P. M.; Zewail, A. H. J. Chem. Phys. 1984 81,4706 .

(47) Vachev, V. D.; Frederick, J. H.; Grishanin, B. A.; Zadkov, V. N. Koroteev, N. I. Chem. Phys. Lett. 1993, 215, 306

(48) Hohlneicher, G.; Müller, M.; Demmer, M.; Lex, J.; Penn, J. H.;

Gan, L.; Loesel, P. D. J. Am. Chem. Soc. 1988, I10, 4483.

(49) (a) Yan, Y. J.; Mukamel, S. J. Chem. Phys. 1986, 85, 5908. (b)

Shan, K.; Yan, Y. J.; Mukamel, S. J. Chem. Phys. 1987, 87, 2021.

(50) Fano, U. Rev. Mod. Phys. 1957, 29, 74.

(51) Feynman, R. P. Phys. Rev. 1951, 84, 108.

(52) (a) Vachev, V. D.; Grishanin, B. A.; Zadkov, V. N. Bull. Russian Acad. Sci. Phys. 1992, 56, 472 (Engl.). (b) Grishanin, B. A.; Vachev, V. D.; Zadkov, V. N. Mol. Cryst. Liq. Cryst. Sci. Technol., Sect B 1992, 3 , 375. (c) Grishanin, B. A. Quantum Electrodynamics for Radiophysicists; Moscow State Univ.: Moscow, 1983 (in Russian).

(53) Fried, L. E.; Mukamel, S. Adv. Chem. Phys. 1993, 84, 435.

(54) Burkert, U.; Allinger, N. L. Molecular Mechanics; American Chemical Society: Washington, DC, 1982; Vol. 177.

(55) Vinter, J.; Davis, A.; Saunders, M. J. Comput-Aided Mol. Des. $1987,1,31$

(56) (a) Allinger, N. L.; Sprague, J. T. J, Am. Chem. soc, 1973, 95, 3893. (b) Kao, J.; Allinger, N. L. J. Am. Chem. Soc. 1977, 99, 975.

(57) (a) Allinger, N. L.; Yuh, Y. H.; Lee, J.-H. J. Am. Chem. Soc. 1989 , 111, 8551. (b) Lee, J.-H.; Allinger, N. L. J. Am. Chem. Soc. 1989, 111 8566.

(58) Hill, T. L. J. Chem. Phys, 1948, 16, 399.

(59) McCoy, A. B.; Sibert, E. L., III. J. Chem. Phys. 1991, 95, 3476

(60) Chiang, W.-Y.; Laane, J. J. Chem. Phys. 1994, 100, 8755.

(61) Bañares, L.; Heikal, A. A.; Zewail, A. H. J. Phys. Chem. 1992 $96,4127$.

(62) Levine, R. D.; Bernstein, R. B. Molecular reaction dynamics and chemical reactivity; Oxford University Press: Oxford, 1987.

(63) (a) Tully, J. C.; Preston, R. K. J. Chem. Phys. 1971, 55, 562. (b)

Tully, J. C. In Dynamics of Molecular Collisions; Miller, W. H., Ed. Plenum: New York, 1976, Part B, Chapter 5, p 217.

(64) Miller, W. H.; George, T. F. J. Chem. Phys. 1972, 56, 5637.

(65) Tully, J, C. J. Chem. Phys. 1990, 93, 1061

(66) (a) Birge, R. R.; Findsen, L. A.; Pierce, B. M. J. Am. Chem. Soc. 1987, 109, 5041. (b) Birge, R. R.; Hubbard, L. M. J. Am. Chem. Soc. 1980, 102,2195 . (c) Birge, R. R.; Hubbard, L. M. Biophys. J, 1981, 34 , 517.

(67) Park, N. S.; Waldeck, D. H. Chem. Phys Lett. 1990, 168, 379.

(68) Warshel, A.; Chu, Z. T.; Hwang, J.-K. Chem. Phys. 1991, 158, 303.

(69) Seidner, L.; Domcke, W. Chem. Phys. 1994, 186, 27.

(70) Saltiel, J.; Waller, A. S.; Sears, D. F., Jr. J. Photochem. Photobiol. A 1992, 65, 29

JP942972X 